\title{
Norming infinitesimals of large fields
}

\author{
H. G. Dales
}

\begin{abstract}
We give a survey of results on norming the infinitesimals of large fields and on constructing discontinuous homomorphisms from Banach algebras of continuous functions; we raise questions that remain from earlier work. The article is expanded from a talk given in Harvard on 27 March 2015 at the conference in honour of the $60^{\text {th }}$ birthday of W. Hugh Woodin. It includes some historical remarks about the first written mathematics of Hugh.
\end{abstract}

\section{Introduction}

It is a pleasure and an honour to be invited to lecture on the theory of discontinuous homomorphisms from Banach algebras of continuous functions, and the closely related question of norming the algebra of infinitesimals of various large fields.

The question whether all algebra norms on the algebra $C(\Omega)$ are equivalent to the uniform norm on $\Omega$, for each compact space $\Omega$, was first raised by Kaplansky long ago $[\mathbf{2 0}]$, and led to much work on the structure of commutative Banach algebras, especially by Bade and Curtis $[\mathbf{3}]$. It was shown independently by myself [5] and by Jean Esterle [13] in two papers that were both submitted for publication in May 1976 that, in the theory ZFC $+\mathrm{CH}$, there are algebra norms that are not equivalent to the uniform norm on $C(\Omega)$ for each infinite, compact space $\Omega$. (The results were summarized in [7].) We both regarded it, with little thought, as obvious that the use of $\mathrm{CH}$ in the proof was a blemish, and that a little more ingenuity would remove the need to appeal to this axiom.

We were stunned to discover that it was a result of W. H. Woodin that this was not the case; there are models of $\mathrm{ZFC}$ in which all algebra norms on each algebra $C(\Omega)$ are equivalent to the uniform norm. We were even more amazed to find that, at the time of this proof, Hugh was an undergraduate at Caltech; there are further comments on this history later.

It is perhaps the case that Kaplansky's question was the first one that appeared to be totally within functional analysis, and yet was shown, to general surprise, to be independent of ZFC.

I first met Hugh in the year 1983-84 when he was one of my TAs for a calculus class that I was teaching at Berkeley. ${ }^{1}$ Subsequently we worked together on two books $[\mathbf{8}, \mathbf{9}]$, and this meant that Hugh came to our house in England quite a few

\footnotetext{
2010 Mathematics Subject Classification. Primary 46H40, 03E35, 03E50.

${ }^{1}$ As a TA, Hugh was initially quite puzzled that there were students at Berkeley who did not understand concepts that were very obvious to him, but he quickly learned about reality.
} 
times. This was great pleasure for us. It was something of a shock to my wife and myself to receive an invitation to come to Harvard for a conference to mark Hugh's $60^{\text {th }}$ birthday: how could this charming young man have reached so mature a level? And now Hugh is a Professor at Harvard: our best congratulations!

Work after 1983 clarified and extended several of the original results, and proofs were presented from a different aspect, which we shall describe here; the main exposition of these results is given in $[\mathbf{6}, \S 5.7]$. Nevertheless, there remain a number of apparently challenging, related open questions; some of these will be mentioned below.

1.1. Basic definitions. We first recall some basic definitions.

The cardinality of a set $S$ is written as $|S|$; the continuum has cardinality c, and so $\mathfrak{c}=2^{\aleph_{0}} ; \mathrm{CH}$ is the continuum hypothesis, i.e., the statement that $\mathfrak{c}=\aleph_{1}$; GCH is the generalized continuum hypothesis, which we shall use just to see that $2^{\aleph_{0}}=\aleph_{1}$ and $2^{\aleph_{1}}=\aleph_{2}$.

Let $A$ be an associative algebra over a field $\mathbb{F}$, always the real field $\mathbb{R}$ or the complex field $\mathbb{C}$. The algebra formed by adjoining an identity to a non-unital algebra $A$ is denoted by $A^{\sharp}$, with $A^{\sharp}=A$ when $A$ already has an identity; the identity of $A^{\sharp}$ is denoted by $e_{A}$. The Jacobson radical of $A$ is denoted by $\operatorname{rad} A ;^{2}$ the algebra $A$ is semi-simple if $\operatorname{rad} A=\{0\}$ and radical if $\operatorname{rad} A=A$.

An element $a$ in an algebra $A$ is nilpotent if $a^{n}=0$ for some $n \in \mathbb{N}$; the set of these elements is denoted by nil $A$. An element $a$ in a complex algebra $A$ is quasi-nilpotent if $z e_{A}-a$ is invertible in the unital algebra $A^{\sharp}$ for each non-zero complex number $z$; the set of these elements is denoted by $\mathfrak{Q}(A)$. It is clear that nil $A \subset \mathfrak{Q}(A)$ and that $\operatorname{rad} A \subset \mathfrak{Q}(A)$; further, $A$ is radical if and only if $\mathfrak{Q}(A)=A$.

An ideal $P$ in an algebra $A$ is a prime ideal if $P$ is a proper ideal and if either $a \in P$ or $b \in P$ whenever $\{a x b: x \in A\}$ is a subset of $P$. In the case where $A$ is commutative, a proper ideal $P$ is prime if and only if either $a \in P$ or $b \in P$ whenever $a b \in P$, and then $A$ is an integral domain if $\{0\}$ is a prime ideal; in this latter case, $A$ has a quotient field, or field of fractions, that consists of the fractions $a / b$, where $a, b \in A$ with $b \neq 0$, taken with the obvious algebraic operations.

A character on a complex algebra $A$ is a homomorphism from $A$ onto $\mathbb{C}$. The set of all characters on $A$ is denoted by $\Phi_{A}$; this is the character space of $A$.

Definition 1.1. An algebra semi-norm on an algebra $A$ is a map $\|\cdot\|: A \rightarrow \mathbb{R}^{+}$ such that:

$$
\|a+b\| \leq\|a\|+\|b\|, \quad\|\alpha a\|=|\alpha|\|a\|, \quad\|a b\| \leq\|a\|\|b\|
$$

for $a, b \in A$ and $\alpha \in \mathbb{F}$; the semi-norm is an algebra norm on $A$ if, further, $\|a\| \neq 0$ for $a \neq 0$. An algebra $A$ is semi-normable, respectively, normable, if there is a non-zero algebra semi-norm, respectively, an algebra norm, on $A$.

A pair $(A,\|\cdot\|)$, where $A$ is a complex algebra, is a Banach algebra if $\|\cdot\|$ is an algebra norm on $A$ and $(A,\|\cdot\|)$ is a Banach space.

\footnotetext{
${ }^{2}$ The general definition of $\operatorname{rad} A$ for non-commutative algebras $A$ is not important for us. However we recall that: a left ideal $I$ in an algebra $A$ is modular if there exists $u \in A$ such that $a-a u \in I(a \in A) ; \operatorname{rad} A$ is defined to be the intersection of all the maximal modular left ideals in $A$, with $A$ taken to be radical when there are no maximal modular left ideals; $\operatorname{rad} A$ is always a two-sided ideal in $A$. See $[6, \S 1.3]$.
} 
Let $A$ be a Banach algebra. We recall that $\operatorname{rad} A$ is always a closed, two-sided ideal in $A$ and that $A / \operatorname{rad} A$ is a semi-simple Banach algebra. All characters $\varphi$ on $A$ are continuous linear functionals, with $\|\varphi\| \leq 1$ [2, Theorem 4.43], and so $\Phi_{A}$ can be regarded as a subset of the closed unit ball $\left(A^{\prime}\right)_{[1]}$ of the dual space $A^{\prime}$ of $A$; of course, $A^{\prime}$ is the Banach space consisting of all continuous linear functionals on the Banach space $A$, and, by the classical Banach-Alaoglu theorem, $\left(A^{\prime}\right)_{[1]}$ is compact with respect to the weak ${ }^{*}$-topology on $A^{\prime}$. Indeed, $\Phi_{A}$ is a locally compact space with respect to this topology.

By the 'spectral radius formula' (given in [2, Theorem 4.23] and [6, Theorem 2.3.8(iii)]), an element $a$ in a Banach algebra $A$ is quasi-nilpotent if and only if $\lim _{n \rightarrow \infty}\left\|a^{n}\right\|^{1 / n}=0$. In the case where $A$ is a commutative Banach algebra, an element $a \in A$ is quasi-nilpotent if and only if $\varphi(a)=0$ for each character $\varphi$, and $\mathrm{SO}$

$$
\operatorname{rad} A=\mathfrak{Q}(A)=\left\{a \in A: \lim _{n \rightarrow \infty}\left\|a^{n}\right\|^{1 / n}=0\right\}=\left\{a \in A: \varphi(a)=0\left(\varphi \in \Phi_{A}\right)\right\} .
$$

Further, the maximal modular ideals in $A$ are exactly the kernels of the characters on $A$, and so all have codimension 1 in $A$. Thus, in this case, the theory simplifies considerably.

A foundation stone of the theory of automatic continuity is the following theorem of Šilov from 1947 [6, Theorem 2.3.3].

Theorem 1.2. Let $A$ and $B$ be Banach algebras, and let $\theta: A \rightarrow B$ be a homomorphism. Suppose that $B$ is commutative and semi-simple. Then $\theta$ is automatically continuous.

There is an introductory account of Banach spaces and Banach algebras that (more than) covers the above theory in [2], and there is a comprehensive account in the monograph $[6]$. In particular, $[6]$ gives many more automatic continuity theorems, some of which will be mentioned below.

Throughout this article, $X$ will often denote a non-empty, completely regular topological space, and then $C(X)$ is the (real) algebra of all continuous, real-valued functions on $X$; the corresponding complex algebra is $C(X, \mathbb{C}){ }^{3}$ Of course, the algebraic operations in these algebras are defined pointwise. The subalgebra of $C(X, \mathbb{C})$ consisting of the bounded functions is denoted by $C^{b}(X, \mathbb{C})$, with $\ell^{\infty}(\mathbb{C})$ for $C^{b}(\mathbb{N}, \mathbb{C})$, the standard Banach space of all bounded sequences on $\mathbb{N}$. The algebra $C^{b}(X, \mathbb{C})$ is a commutative Banach algebra with respect to the uniform norm $|\cdot|_{X}$ on $X$, where

$$
|f|_{X}=\sup \{|f(x)|: x \in X\} \quad\left(f \in C^{b}(X, \mathbb{C})\right),
$$

and $C^{b}(X, \mathbb{C})$ is semi-simple because it is clear that there are no non-zero quasinilpotent elements in $C^{b}(X, \mathbb{C})$. It is standard that the algebra $C^{b}(X, \mathbb{C})$ can be identified by an isometric algebra isomorphism with $C(\beta X, \mathbb{C})$, where $\beta X$ is the Stone-Čech compactification of the space $X ;^{4}$ in particular, we identify $\ell^{\infty}(\mathbb{C})$

\footnotetext{
${ }^{3}$ A Hausdorff topological space $X$ is completely regular if, for each $x \in X$ and each open neighbourhood $U$ of $x$, there exists $f \in C(X)$ with $f(x)=1$ and $f(y)=0(y \in X \backslash U)$. Every locally compact space is completely regular.

${ }^{4}$ This is a special case of the Gel'fand representation theorem, given in [6, Theorem 2.3.25], for example. It is also immediate from the definition of the Stone-Cech compactification, $\beta X$.
} 
with the space $C(\beta \mathbb{N}, \mathbb{C})$. We shall write $X^{*}$ for the growth of the space $X$ in $\beta X$, so that $X^{*}=\beta X \backslash X$; in particular, we have the notation $\mathbb{N}^{*}=\beta \mathbb{N} \backslash \mathbb{N}$.

In the case where $X$ is locally compact (always Hausdorff), we take $C_{0}(X, \mathbb{C})$ to be the closed ideal in $C^{b}(X, \mathbb{C})$ consisting of the functions that vanish at infinity, and set $c_{0}(\mathbb{C})=C_{0}(\mathbb{N}, \mathbb{C})$, so that $c_{0}(\mathbb{C})$ is the space of all null-sequences; $C(\Omega, \mathbb{C})$ is a complex, commutative, unital Banach algebra for the uniform norm whenever $\Omega$ is a non-empty, compact space.

The basic examples to have in mind are the following: $C(\mathbb{I}, \mathbb{C})$, where $\mathbb{I}=[0,1]$ is the closed unit interval in $\mathbb{C} ; \ell^{\infty}(\mathbb{C})$; and $c_{0}(\mathbb{C})$. Perhaps surprisingly, the most complicated of these is $C(\mathbb{I}, \mathbb{C})$.

Let $\Omega$ be a non-empty, compact space, and take $x \in \Omega$. Then

$$
M_{x}=\{f \in C(\Omega, \mathbb{C}): f(x)=0\},
$$

and $J_{x}$ is the set of functions in $C(\Omega, \mathbb{C})$ that vanish on a neighbourhood of $x$, the neighbourhood depending on the function. Clearly $J_{x}=M_{x}$ whenever $x$ is isolated in $\Omega$, and $J_{x}$ is always a dense ideal in $M_{x}$ for each $x \in \Omega$. It is standard and easy to see (see [2, Example 4.49] or [6, Theorem 4.2.1(i)]) that $\left\{M_{x}: x \in \Omega\right\}$ is the family of all maximal ideals in $C(\Omega, \mathbb{C})$, equivalently, every character on $C(\Omega, \mathbb{C})$ has the form

for some $x \in \Omega$.

$$
\varepsilon_{x}: f \mapsto f(x), \quad C(\Omega, \mathbb{C}) \rightarrow \mathbb{C},
$$

Definition 1.3. A radical homomorphism from an algebra $A$ is a non-zero homomorphism from $A$ into a commutative, radical Banach algebra.

Let $\Omega$ be a compact space, and take $x \in \Omega$.

Suppose that $\theta: M_{x} \rightarrow B$ is a non-zero homomorphism into a commutative Banach algebra $B$ such that $\theta \mid J_{x}=0$. For each $\psi \in \Phi_{B}$, we have $(\psi \circ \theta) \mid J_{x}=0$, and so $(\psi \circ \theta) \mid M_{x}=0$ because $\psi \circ \theta$ is continuous and $\overline{J_{x}}=M_{x}$. Thus $\theta\left(M_{x}\right) \subset\left\{\operatorname{ker} \psi: \psi \in \Phi_{B}\right\}=\operatorname{rad} B$, and so $\theta$ is a radical homomorphism.

Conversely, suppose that $\theta: M_{x} \rightarrow B$ is a homomorphism into a commutative, radical Banach algebra $B$, and take $f \in J_{x}$. Then there exists $g \in M_{x}$ with $f g=f$, and so $\theta(f)\left(e_{B}-\theta(g)\right)=0$. Since $B$ is a radical algebra, $e_{B}-\theta(g)$ is invertible in $B^{\sharp}$, and so $\theta(f)=0$. Thus $\theta \mid J_{x}=0$.

It follows that a non-zero homomorphism $\theta$ from an algebra $M_{x}$ into a commutative Banach algebra is a radical homomorphism if and only if $\theta \mid J_{x}=0$. Thus there is a non-zero homomorphism $\theta$ from $M_{x}$ into a commutative, radical Banach algebra $B$ if and only if there is an ideal $I=\operatorname{ker} \theta$ in $C(\Omega, \mathbb{C})$ with $J_{x} \subset I \subsetneq M_{x}$ such that the induced homomorphism from $M_{x} / I$ into $B$ is an injection, and hence such that $M_{x} / I$ is normable, i.e., if and only if $M_{x} / J_{x}$ is semi-normable.

1.2. Kaplansky's problem. Let $\Omega$ be a non-empty, locally compact space, and take $\|\cdot\|$ to be an algebra norm on $C_{0}(\Omega, \mathbb{C})$. Then Kaplansky proved in $[\mathbf{2 0}]$ that

$$
|f|_{\Omega} \leq\|f\| \quad\left(f \in C_{0}(\Omega)\right) ;
$$

see [6, Theorem 4.2.3(ii)]. He then asked whether the two norms are always equivalent, in the sense that there is a constant $C>0$ such that

$$
\|f\| \leq C|f|_{\Omega} \quad\left(f \in C_{0}(\Omega)\right) .
$$


Of course, it is an immediate consequence of Banach's isomorphism theorem [2, Corollary 3.41$]$ that this is true whenever the algebra norm $\|\cdot\|$ is complete. Also, by Theorem 1.2, it is true whenever the completion of $\left(C_{0}(\Omega),\|\cdot\|\right)$ is semi-simple.

Suppose that $\|\cdot\|$ is a norm on $C_{0}(\Omega, \mathbb{C})$ that is not equivalent to the uniform norm, and take $B$ to be the commutative Banach algebra that is the completion of $\left(C_{0}(\Omega, \mathbb{C}),\|\cdot\|\right)$. Then the embedding of $\left(C_{0}(\Omega, \mathbb{C}),|\cdot|_{\Omega}\right)$ into $B$ is discontinuous. Conversely, suppose that $\theta: C_{0}(\Omega, \mathbb{C}) \rightarrow B$ is a discontinuous homomorphism into a Banach algebra $B$. Then the formula

$$
\|f\|=\max \left\{|f|_{\Omega},\|\theta(f)\|\right\} \quad\left(f \in C_{0}(\Omega, \mathbb{C})\right)
$$

defines an algebra norm $\|\cdot\|$ on $C_{0}(\Omega, \mathbb{C})$ that is not equivalent to the uniform norm. Thus the following theorem [6, Theorem 4.2.3(iii)] is a re-wording of Kaplansky's result.

THeOREM 1.4. Let $\Omega$ be a non-empty, locally compact space. Then the following are equivalent:

(a) there is an algebra norm on $C_{0}(\Omega, \mathbb{C})$ that is not equivalent to the uniform norm;

(b) there is a discontinuous homomorphism from $C_{0}(\Omega, \mathbb{C})$ into some Banach algebra.

In fact, Kaplansky's problem has usually been considered in the setting of clause (b), above. To ease future discussion, it is convenient to state an axiom called $\mathrm{NDH}$ ('no discontinuous homomorphisms'); this was first specifically formulated by Solovay.

NDH: For each compact space $\Omega$, each homomorphism from $C(\Omega, \mathbb{C})$ into a Banach algebra is continuous.

Thus Kaplansky's problem asks whether NDH is true in ZFC. We note that there are many (non-commutative) Banach algebras $A$ such that all homomorphisms from $A$ into every Banach algebra are automatically continuous; see $\S 4.3$ and $[6]$.

Several claims of a positive solution of Kaplansky's problem were made, and they were investigated by Bade and Curtis around 1960 in a seminar at Yale; all proved to be erroneous. However, the request of Charles Rickart to these younger colleagues to investigate the claims led to their interest in Kaplansky's question, and hence to the seminal paper [3], so the false claims did play an important role in the story.

The main theorem of the paper of Bade and Curtis describes in detail the structure of a homomorphism from a general algebra of the form $C_{0}(\Omega, \mathbb{C}$ ) (and, in fact, from more general 'strong Ditkin algebras') into a commutative Banach algebra. We give an abbreviated and informal form of this theorem here; a full version is in [6, Theorem 5.4.22] and [8, Theorem 1.6].

THEOREM 1.5. Let $\Omega$ be a non-empty, compact space, and suppose that $\theta$ is a homomorphism from $C(\Omega, \mathbb{C})$ into a Banach algebra $A$. Then either $\theta$ is continuous or there is a non-empty, finite subset $\left\{x_{1}, \ldots, x_{n}\right\}$ of $\Omega$ such that $\theta \mid B$ is continuous for a certain dense, unital subalgebra $B$ of $C(\Omega, \mathbb{C})$ such that $B$ contains the ideal $J_{x_{1}} \cap \cdots \cap J_{x_{n}}$. Further, in the latter case, there is a radical homomorphism from $M_{x}$ for some $x \in \Omega$. 
Corollary 1.6. Let $\Omega$ be a non-empty, compact space. Then there is an algebra norm on $C(\Omega, \mathbb{C})$ that is not equivalent to the uniform norm if and only if there exists $x \in \Omega$ such that $J_{x} \subsetneq M_{x}$ and the quotient space $M_{x} / J_{x}$ is seminormable.

Thus the study of Kaplansky's problem for $C(\Omega, \mathbb{C})$ reduces to the question of the existence of $x \in \Omega$ and an ideal $I$ in $C(\Omega, \mathbb{C})$ such that $J_{x} \subset I \subsetneq M_{x}$ and $M_{x} / I$ is normable.

We slightly reformulate and extend the above in the following convenient setting: now $\Omega$ is a non-empty, locally compact, non-compact space, and $\theta$ is an assumed discontinuous homomorphism from $C_{0}(\Omega)$ into a Banach algebra $B$. The continuity ideal, denoted by $\mathcal{I}(\theta)$, of $\theta$ is defined to be the largest ideal $I$ of $C_{0}(\Omega)$ such that $\theta \mid I$ is continuous. ${ }^{5}$ It follows from the theorem of Bade and Curtis and extensions thereof by Esterle [12] and by Sinclair [25] that the ideals $\operatorname{ker} \theta$ and $\mathcal{I}(\theta)$ are always intersections of prime ideals and that $\mathcal{I}(\theta)=\operatorname{ker} \theta$ whenever $B$ is a radical Banach algebra. Thus we obtain a further reformulation of Kaplansky's problem, as follows; this is the form in which it will be discussed.

THEOREM 1.7. Let $\Omega$ be a non-empty, compact space. Then the following are equivalent:

(a) there is an algebra norm on $C(\Omega, \mathbb{C})$ that is not equivalent to the uniform norm;

(b) there is a maximal ideal $M$ and a prime ideal $P$ in $C(\Omega, \mathbb{C})$ with $P \subsetneq M$ such that the algebra $M / P$ is normable.

(c) there is a maximal ideal $M$ and a prime ideal $P$ in $C(\Omega, \mathbb{C})$ with $P \subsetneq M$ and an embedding of $M / P$ into a commutative, radical Banach algebra.

We note that, for each infinite, compact space $\Omega$, the algebra $C(\Omega, \mathbb{C})$ contains a maximal ideal $M_{x}$ and a prime ideal $P$ with $J_{x} \subset P \subsetneq M_{x}$ such that $\left|M_{x} / P\right|=\mathfrak{c}$ for some $x \in \Omega$.

The main theorem proved in [5] and [12] (in different ways and by long calculations) was the following.

THEOREM 1.8. (CH) Let $\Omega$ be an infinite compact space. Then there is a maximal ideal $M$, a prime ideal $P$ in $C(\Omega, \mathbb{C})$ with $P \subsetneq M$, and a homomorphism with kernel $P$ from $M$ into a certain radical Banach algebra, and hence the algebra $M / P$ is normable.

Thus $\mathrm{CH}$ implies $\neg \mathrm{NDH}$.

The above suggests a formulation of our question in the language of ordered fields, and we shall consider such a version in $\S 3.2$.

\section{Ordered groups and fields}

We first recall some background in the theory of ordered groups and fields. All the results of this section are taken from $[\mathbf{9}]$.

\footnotetext{
${ }^{5}$ There are more general definitions in $[6]$.
} 
2.1. Ordered sets. Let $S$ be a non-empty set. A strict partial order on $S$ is a binary relation $<$ on $S$ such that: (i) if $a<b$ and $b<c$ in $S$, then $a<c$; (ii) $a \nless a$ for each $a \in S$. The order is a total order if, for each $a, b \in S$, either $a<b$ or $a=b$ or $b<a$. A partially ordered set is a pair $(P,<)$, where $S$ is a non-empty set and $<$ is a strict partial order on $S$. We set $a \leq b$ if $a<b$ or $a=b$ in $S$. A totally ordered set $S$ is well-ordered if each non-empty subset of $S$ has a minimum element.

We shall require the following examples later.

EXAMPLES 2.1. (i) Let $R$ be a commutative, radical algebra, and consider the set $S=R \backslash\{0\}$. For $a, b \in S$, set $a \ll b$ if $a \in b R$. Then $\ll$ is a strict partial order on $S$, called the divisibility order.

(ii) Let $f, g \in \mathbb{N}^{\mathbb{N}}$, and then define $f<_{\mathcal{F}} g$ if there exists $n_{0} \in \mathbb{N}$ such that $f(n)<g(n) \quad\left(n \geq n_{0}\right)$ and $f \ll_{\mathcal{F}} g$ if $g(n)-f(n) \rightarrow \infty$ as $n \rightarrow \infty$. We see that $<_{\mathcal{F}}$ and $\ll_{\mathcal{F}}$ are strict partial orders on $\mathbb{N}^{\mathbb{N}}$, called the Fréchet order and the strong Fréchet order, respectively.

Let $(S,<)$ and $(T,<)$ be partially ordered sets. A map $\pi: S \rightarrow T$ is isotonic, respectively, anti-isotonic, if $\pi(a)<\pi(b)$, respectively, $\pi(b)<\pi(a)$, whenever $a<b$ in $S$; the map $\pi$ is an order-isomorphism if it is a bijection such that $\pi$ and $\pi^{-1}$ are isotonic.

Let $(S, \leq)$ be a totally ordered set. A subset $T$ of $S$ is: order-dense if, for each $s_{1}, s_{2} \in S$ with $s_{1}<s_{2}$ there exists $t \in T$ with $s_{1} \leq t \leq s_{2}$; cofinal in $S$ if, for each $s \in S$, there exists $t \in T$ with $s \leq t$; coinital in $S$ if, for each $s \in S$, there exists $t \in T$ with $t \leq s$. The weight, $w(S)$, of $S$ is the minimum cardinality of an orderdense subset; the cofinality and coinitiality of $S$ are the minimum cardinalities $\kappa$ and $\lambda$ of a strictly increasing and strictly decreasing, respectively, sequence whose range is cofinal and coinitial, respectively, in $S$; we then write

$$
\operatorname{cof} S=\kappa \quad \text { and } \quad \operatorname{coi} S=\lambda .
$$

Take $S_{1}$ and $S_{2}$ to be subsets of $S$. Then $S_{1} \ll S_{2}$ if $s_{1}<s_{2}$ whenever $s_{1} \in S_{1}$ and $s_{2} \in S_{2}$.

Of course $(\mathbb{R}, \leq)$ is a totally ordered set of cardinality $|\mathbb{R}|=\mathfrak{c}$ and weight $w(\mathbb{R})=\aleph_{0}$; the subset $\mathbb{Q}$ of rational numbers is an order-dense subset.

2.2. Ordered groups. Let $G=(G,+)$ be an abelian group with identity 0 . Then $G$ is divisible if, for each $x \in G$ and each $n \in \mathbb{N}$, there exists $y \in G$ with $n y=x$ A map $\psi: G \rightarrow H$ between two groups $G$ and $H$ is a group morphism if

$$
\psi(x+y)=\psi(x)+\psi(y) \quad(x, y \in G) .
$$

Now suppose that $G$ is a group with a partial order $\leq$. Then $(G,+, \leq)$ is an ordered group if $x+z<y+z$ whenever $x<y$ in $G$ and $z \in G$. We then write $G^{+}=\{x \in G: x \geq 0\}$ for the positive elements of $G$. Suppose further that $(G, \leq)$ is a lattice; for example, $\mathbb{R}^{S}$ is an ordered group that is a lattice with respect to the usual operations for each non-empty set $S$. Then $x^{+}=x \vee 0$ and $x^{-}=x \wedge 0$ for $x \in G$, so that $x=x^{+}+x^{-}(x \in G)$, and we define $|x|=x^{+}-x^{-}(x \in G)$. A subset $S$ of $G$ is absolutely convex if $x \in S$ whenever $|x| \leq|y|$ for some $y \in S$. Further, an ordered group $(G,+, \leq)$ is a totally ordered group if $\leq$ is a total order on $G$; in this case $(G, \leq)$ is a lattice. The base number of such a group is

$$
\delta(G)=\operatorname{coi}\left(G^{+} \backslash\{0\}\right) .
$$


Let $G$ be a totally ordered group. For $x, y \in G$, set $x=\mathbf{O}(y)$ if $|x| \leq n|y|$ for some $n \in \mathbb{N}$, and $x \sim y$ if $x=\mathbf{O}(y)$ and $y=\mathbf{O}(x)$. The set

$$
\Gamma_{G}=(G \backslash\{0\}) / \sim
$$

is the value set of $G$, and the quotient map $v$ is the archimedean valuation; set $v(x) \leq v(y)$ if $x=\mathbf{O}(y)$. Then $\left(\Gamma_{G}, \leq\right)$ is a totally ordered set.

Two totally ordered groups $G$ and $H$ are isomorphic if there is a group morphism from $G$ onto $H$ that is also an order-isomorphism.

2.3. Ordered fields. A field is taken to be a field over $\mathbb{R}$, and all isomorphisms between our fields must be $\mathbb{R}$-linear; the identity of a field is usually denoted by 1 .

Let $K$ be a field, and suppose that $(K,+, \leq)$ is a totally ordered group. Then $(K,+, \cdot, \leq)$ is an ordered field if $a b=a \cdot b>0$ whenever $a, b>0$ in $K$ and if $\alpha a>0$ whenever $a>0$ in $K$ and $\alpha>0$ in $\mathbb{R}$.

A convex subgroup of $K$ is a subgroup $I$ of $(K,+)$ such that $1 \in I$ and $I$ is an absolutely convex set.

An ordered field $K$ is real-closed if every positive element is a square and every polynomial over $K$ of odd degree has a root; equivalently, $K$ is real-closed if the complexification $K(\mathrm{i})$ of $K$ is algebraically closed. The Artin-Schreier theorem says that every ordered field has an algebraic extension to a real-closed field of the same cardinality, and so, when seeking to show that all ordered fields of a given cardinality are normable, it is sufficient to consider real-closed fields. Of course the real line $\mathbb{R}$ is the proto-typical real-closed field.

Definition 2.2. Let $K$ be an ordered field with identity 1 , and let $a \in K$. Then: $a$ is an infinitesimal if $|a| \leq(1 / n) 1$ for all $n \in \mathbb{N} ; a$ is finite if $|a| \leq n 1$ for some $n \in \mathbb{N}$; and $a$ is infinitely large if $|a| \geq n 1$ for all $n \in \mathbb{N}$.

Thus 0 is an infinitesimal in each ordered field; in a large field there are non-zero infinitesimals. Suppose that $a>0$ and $a$ is an infinitesimal. Then $1 / a$ is infinitely large.

We write $K^{\sharp}$ and $K^{\circ}$ for the algebras of finite elements and of infinitesimals, respectively, in an ordered field $K$. Thus $K^{\sharp}$ is formed by adjoining an identity to the algebra $K^{\circ}$, and so is $\left(K^{\circ}\right)^{\sharp}$ in the previous notation.

Let $K$ be an ordered field with value set $\Gamma_{K}$. Define + on $\Gamma_{K}$ by

$$
v(a)+v(b)=v(a b) \quad(a, b \in K \backslash\{0\}) .
$$

Then the operation + is well-defined, and $\left(\Gamma_{K},+, \leq\right)$ is a totally ordered group, called the value group of $K$. Clearly

$$
K^{\circ}=\{a \in K: v(a)>0\} \quad \text { and } \quad K^{\sharp}=\{a \in K: v(a) \geq 0\} .
$$

The value group $\Gamma_{K}$ is divisible whenever $K$ is real-closed.

For example, let $X$ be a non-empty, completely regular topological space, and let $M$ be a maximal ideal in the real algebra $C(X)$. Then it is easily checked that the quotient algebra $C(X) / M$ is a real-closed ordered field; we shall prove a more general result below. These are the hyper-real fields; see the seminal text of Gillman and Jerison [17, $\S \S 5.6,13.4]$ and [9, Definition 4.17].

In particular, suppose that $X$ is discrete; it is just a set. Then we obtain realclosed ordered fields that are isomorphic to fields of the form $\mathbb{R}^{\kappa} / M$, where $\kappa$ is 
a cardinal and $M$ is a maximal ideal in the real algebra $\mathbb{R}^{\kappa}$. By the Gel'fandKolmogorov theorem, ${ }^{6}$ maximal ideals in $\mathbb{R}^{\kappa}$ correspond to ultrafilters on $\kappa$, and so these fields are called ultrapowers. We recall that a filter $\mathcal{F}$ on a non-empty set $S$ is a non-empty family of subsets of $S$ with the following properties: $\emptyset \notin \mathcal{F}$; $F \cap G \in \mathcal{F}$ whenever $F, G \in \mathcal{F} ; G \in \mathcal{F}$ whenever $G$ is a subset of $S$ and $G \supset F$ for some $F \in \mathcal{F}$. An ultrafilter on $S$ is a maximal filter when the family of filters on $S$ is ordered by inclusion; equivalently, a filter $\mathcal{U}$ on $S$ is an ultrafilter if $F \in \mathcal{U}$ or $G \in \mathcal{U}$ whenever $F$ and $G$ are subsets of $S$ and $F \cup G \in \mathcal{U}$. Thus every ultrapower is a hyper-real field. These ultrapowers are usually written as

$$
\mathbb{R}^{\kappa} / \mathcal{U} \text { or }\left(\mathbb{R}^{\kappa} / \mathcal{U},<\mathcal{U}\right),
$$

where $\mathcal{U}$ is the ultrafilter on $\kappa$ corresponding to the maximal ideal $M$. Here the equivalence class containing $f \in \mathbb{R}^{\kappa}$ is

$$
[f]_{\mathcal{U}}=\left\{g \in \mathbb{R}^{\kappa}:\{\sigma<\kappa: g(\sigma)=f(\sigma)\} \in \mathcal{U}\right\}
$$

we set $f<_{\mathcal{U}} g$ in $\mathbb{R}^{\kappa}$ if $\{\sigma<\kappa: f(\sigma)<g(\sigma)\} \in \mathcal{U}$, and $[f]_{\mathcal{U}}<_{\mathcal{U}}[g]_{\mathcal{U}}$ when $f<_{\mathcal{U}} g$. Thus $<\mathcal{U}$ is a total order on $\mathbb{R}^{\kappa} / \mathcal{U}$.

The classic text on ultrafilters and ultrapowers is Comfort and Negrepontis [4]. There is a study of a generalization of ultrafilters to $z$-ultrafilters on a completely regular space $X$ in $[\mathbf{1 7}]$ and $[\mathbf{6}]$, and then the $z$-ultrafilters on $X$ correspond to the points of $\beta X$.

2.4. Hahn groups and fields. We now give a construction of some groups $\mathfrak{F}(S)$ and $\mathfrak{F}_{(1)}(S)$ and some fields $\mathfrak{F}(G)$ and $\mathfrak{F}_{(1)}(G)$; for more general constructions, see $[\mathbf{9}$, Chapters 1 and 2].

Let $S$ be a set, and take $f \in \mathbb{R}^{S}$. Then $\operatorname{supp} f=\{s \in S: f(s) \neq 0\}$.

Let $S$ be a totally ordered set. Then

$$
\mathfrak{F}(S)=\left\{f \in \mathbb{R}^{S}: \operatorname{supp} f \text { is well-ordered }\right\}
$$

and

$$
\mathfrak{F}_{(1)}(S)=\{f \in \mathfrak{F}(S): \operatorname{supp} f \text { is countable }\} .
$$

It is clear that both $\mathfrak{F}(S)$ and $\mathfrak{F}_{(1)}(S)$ are real-linear subspaces of $\mathbb{R}^{S}$, and so they are divisible groups with respect to the pointwise addition of functions. For $f \in \mathfrak{F}(S)$ with $f \neq 0$, set

$$
v(f)=\inf \operatorname{supp} f
$$

and set $f>0$ in $\mathfrak{F}(S)$ if $v(f)>0$. Then $v$ is the Hahn valuation on $\mathfrak{F}(S)$, and $(\mathfrak{F}(S),+, \leq)$ is a totally ordered group, called the Hahn group of $S$. The value set of $(\mathfrak{F}(S),+, \leq)$ and of $\left(\mathfrak{F}_{(1)}(S),+, \leq\right)$ is $S$ itself, and the Hahn valuation agrees with the previous archimedean valuation.

The Hahn embedding theorem [9, Theorem 1.35] gives a certain 'universal property' of the Hahn groups among totally ordered, divisible groups; we shall not use this theorem.

Now suppose that $G$ is a totally ordered group. Then we can define a product on the linear space $\mathfrak{F}(G)$ : for $f, g \in \mathfrak{F}(G)$, set

$$
(f \star g)(t)=\sum\{f(r) g(s): r, s \in G, r+s=t\} \quad(t \in G) .
$$

\footnotetext{
${ }^{6}$ For more general versions of this theorem, see [6, Theorem 4.2.12] or [17, $\left.\S 7.3\right]$
} 
In fact, the above sum is always a finite sum, ${ }^{7}$ and $f \star g \in \mathfrak{F}(G)$; in the case where $f, g \in \mathfrak{F}_{(1)}(G)$, then also $f \star g \in \mathfrak{F}_{(1)}(G)$. It is now easy to see that $(\mathfrak{F}(G), \star)$ is an algebra over $\mathbb{R}$ and that $\mathfrak{F}_{(1)}(G)$ is a subalgebra of $\mathfrak{F}(G)$. The algebra $(\mathfrak{F}(G), \star)$ is called the Hahn algebra over $G$; the valuation $v$ recovers the value group $G$ of $(\mathfrak{F}(G), \star)$.

The main theorem in this setting is that $(\mathfrak{F}(G), \star)$ is in fact an ordered field, and that $\mathfrak{F}_{(1)}(G)$ is a subfield of $\mathfrak{F}(G)$; a theorem of Saunders MacLane shows that $\mathfrak{F}(G)$ and $\mathfrak{F}_{(1)}(G)$ are real-closed fields whenever $G$ is a divisible group. For these theorems, see [9, Theorem 2.15].

The Kaplansky embedding theorem [9, Theorem 2.17] gives a certain 'universal property' of the Hahn fields $(\mathfrak{F}(G), \star)$ among real-closed ordered fields; a closely related result is Kaplansky's isomorphism theorem [6, Corollary 1.7.36], which is expressed in the language of 'maximal, complex valuation algebras'.

It was Jean Esterle who recognized the importance of the Hahn algebra in this area, and also gave much shorter proofs of some very classical theorems; see [16], for example.

2.5. Formal power series and Allan's theorem. Here is the most elementary, non-trivial example of a Hahn field. For $m \in \mathbb{Z}$, we denote by $\delta_{m}$ the function on $\mathbb{Z}$ such that $\delta_{m}(m)=1$ and $\delta_{m}(n)=0$ for $n \in \mathbb{Z}$ with $n \neq m$.

Consider the totally ordered group $(\mathbb{Z},+)$. Then $\mathfrak{F}(\mathbb{Z})$ consist of the formal Laurent series

$$
a=\sum_{n=n_{0}}^{\infty} \alpha_{n} \delta_{n}
$$

where $n_{0} \in \mathbb{Z}$ (with $n_{0}$ possibly negative) and $\alpha_{n} \in \mathbb{R}\left(n \geq n_{0}\right)$, and multiplication is defined by requiring that $\delta_{m} \star \delta_{n}=\delta_{m+n}(m, n \in \mathbb{Z}){ }^{8}$ The Hahn valuation $v$ is given by

$$
v(a)=\min \left\{n \in \mathbb{Z}: \alpha_{n} \neq 0\right\},
$$

and $\mathfrak{F}(\mathbb{Z})^{\sharp}=\{a: v(a) \geq 0\}$ is clearly identified with

$$
\mathfrak{F}\left(\mathbb{Z}^{+}\right)=\left\{a=\sum_{n=0}^{\infty} \alpha_{n} X^{n}: \alpha_{n} \in \mathbb{R}\left(n \in \mathbb{Z}^{+}\right)\right\},
$$

the algebra of formal power series in one variable. Here $\mathbb{Z}^{+}=\{n \in \mathbb{Z}: n \geq 0\}$. This algebra is often denoted by $\mathbb{R}[[X]]$; its complex version is $\mathbb{C}[[X]]$, and we shall denote this latter algebra here just by $\mathfrak{F}$, writing $\mathfrak{F}^{\circ}$ for its unique maximal ideal.

The algebra $\mathfrak{F}$ is a so-called Fréchet algebra with respect to the topology of coordinatewise convergence $\tau_{c}$, and $\tau_{c}$ is the unique topology with respect to which $\mathfrak{F}$ is a Fréchet algebra [6, Theorem 4.6.1]. It is easy to see that $\mathfrak{F}$ is not a Banach algebra with respect to any norm, but it is certainly not obvious whether or not $\mathfrak{F}$ is normable. But, in a significant break-through, this was shown to be the case by Graham Allan in 1972 [1]. This theorem is given as [6, Theorem 5.7.1]; it uses the algebraic extension methods of $[\mathbf{6}, \S 1.7]$ and an obvious 'framework map', namely $n \mapsto a^{n}, \mathbb{N} \rightarrow A$, for a suitable element $a$ in a Banach algebra $A$, and was an avatar for later embedding theorems.

\footnotetext{
${ }^{7}$ More precisely, the sum has only finitely many non-zero terms. This is because there is no strictly decreasing, infinite sequence in the well-ordered set that is $\operatorname{supp} f$.

${ }^{8}$ Thus these formal series correspond exactly to the usual Laurent series of elementary complex analysis.
} 
Definition 2.3. Let $A$ be a commutative Banach algebra, and let $a \in A$. Then $a$ has finite closed descent if there exists $n \in \mathbb{N}$ such that

$$
a^{n} \in \overline{a^{n+1} A} \backslash a^{n+1} A ;
$$

$a$ has finite closed descent $k$ if the minimum such $n \in \mathbb{N}$ is $k$.

The main theorem of Allan is the following.

Theorem 2.4. Let $A$ be a commutative, unital Banach algebra, and let $a \in A$. Then there is a unital embedding $\theta: \mathfrak{F} \rightarrow A$ with $\theta(X)=a$ if and only if $a \in \operatorname{rad} A$ and a has finite closed descent in $A$.

There are many commutative, radical Banach algebras that contain elements of finite closed descent. For example, let $\mathcal{V}$ be the Banach space $L^{1}(\mathbb{I})$, and set

$$
(f \star g)(s)=\int_{0}^{s} f(s-t) g(t) \mathrm{d} t \quad(s \in \mathbb{I})
$$

for $f, g \in \mathcal{V}$. Then it is easy to check [6, Theorem 4.7.40] that $\mathcal{V}$ is a commutative, radical Banach algebra; it is called the Volterra algebra. Allan noted in [1] that there are elements of finite closed descent in $\mathcal{V}^{\sharp}$, and hence that there is a unital embedding of $\mathfrak{F}$ into $\mathcal{V} \sharp$. It follows that $\mathfrak{F}$ is normable.

2.6. Prime ideals in algebras $C(X)$ and super-real fields. Let $X$ be a non-empty, completely regular topological space. There are substantial, classic studies of the family of prime ideals in the algebras $C(X)$. The starting point is [17, Chapter 14]; this study is taken up in [9].

The following notion is surprisingly important in the study of ideals in the algebras $C(X)$; see [17], for example. The zero set of $f \in C(X)$ is

$$
\mathbf{Z}(f)=\{x \in X: f(x)=0\}=f^{-1}(\{0\}) .
$$

An ideal $I$ in $C(X)$ is a $z$-ideal if $f \in I$ whenever $\mathbf{Z}(f)=\mathbf{Z}(g)$ for some $g \in I$.

Let $P$ be a prime ideal in $C(X)$. Then there are two basic facts. First, $P$ is contained in a unique maximal ideal of $C(X)$, and, second, the set of primes in $C(X)$ containing $P$ forms a totally ordered family with respect to inclusion. Each prime ideal contains a minimal prime ideal. A prime $z$-ideal is a prime ideal that is also a $z$-ideal. There are prime ideals that are not prime $z$-ideals, but every minimal prime ideal is a prime $z$-ideal.

Let $\Omega$ be a non-empty, compact space, and take a prime ideal $P$ in $C(\Omega, \mathbb{C})$. Then there exists $x \in \Omega$ such that $J_{x} \subset P \subset M_{x}$.

Sometimes the ideal $J_{x}$ in $C(\Omega, \mathbb{C})$ is itself a prime ideal; this is the case for each $x \in \beta \mathbb{N}$, and now $J_{x}$ is the unique minimum prime ideal contained in $M_{x}$, and the prime ideals in $M_{x}$ form one totally ordered family. The point $x \in \beta \mathbb{N}$ corresponds to an ultrafilter $\mathcal{U}$ on $\mathbb{N}$, and the quotient algebra $M_{x} / J_{x}$ is naturally identified with the algebra $\left(\mathbb{R}^{\omega} / \mathcal{U}\right)^{\circ}$ of infinitesimals in the field that is the ultrapower $\mathbb{R}^{\omega} / \mathcal{U}$; we shall consider free ultrafilters, corresponding to points of $\mathbb{N}^{*} .{ }^{9}$

However, sometimes $J_{x}$ is not a prime ideal in $C(\Omega, \mathbb{C})$; for example, this is the case for $C(\mathbb{I}, \mathbb{C})$ and each $x \in \mathbb{I}$, and then the family of prime ideals contained in $M_{x}$ is a (very complicated) tree.

\footnotetext{
${ }^{9}$ In fact, each ideal $J_{x}$ in an algebra $C(\Omega, \mathbb{C})$ is a prime ideal if and only if $\Omega$ is an $F$-space, which means that $\bar{U} \cap \bar{V}=\emptyset$ whenever $U$ and $V$ are open $F_{\sigma}$-sets with $U \cap V=\emptyset$.
} 
Definition 2.5. Let $X$ be a non-empty, completely regular topological space, and let $P$ be a prime ideal in $C(X)$. Then $A_{P}$ is the quotient algebra $C(X) / P$, and $K_{P}$ is its quotient field.

Certainly $A_{P}$ is an integral domain, and so $A_{P}$ does have a quotient field, $K_{P}$. It is a basic, if elementary, fact that $A_{P}$ is a totally ordered, commutative, unital algebra [9, Theorem 4.14]. Indeed, take $a \in A_{P}$, say $a=\pi_{P}(f)$ for $f \in C(X)$, where $\pi_{P}$ denotes the quotient map. Then $f=f^{+}+f^{-}$, and clearly $f^{+} f^{-}=0$, so that either $f^{+} \in P$ or $f^{-} \in P$. Hence either $a \geq 0$ or $a \leq 0$ in the quotient order $\leq$ on $A_{P}$. Thus $\left(A_{P}, \leq\right)$ is a totally ordered set. Clearly $a+c<b+c$ whenever $a, b, c \in A_{P}$ with $a<b$, and $a b>0$ whenever $a, b \in A_{P}$ with $a, b>0$. Finally, $\alpha a>0$ in $A_{P}$ whenever $\alpha>0$ in $\mathbb{R}$ and $a>0$ in $A_{P}$. It follows easily that $K_{P}$ is an ordered field in the above sense, and that $A_{P} \subset K_{P}^{\sharp}$.

Definition 2.6. Let $K$ be an ordered field. Then $K$ is a super-real field if $K$ is not isomorphic to $\mathbb{R}$ and $K$ is isomorphic to an ordered field $K_{P}$, where $P$ is prime ideal in $C(X)$ for some completely regular space $X$.

Super-real fields are the topic of the monograph [9].

Clearly every hyper-real field is a super-real field. The key fact that superreal fields are real-closed is proved in [9, Theorem 4.27], somewhat extending the classical fact that hyper-real fields are real-closed [17, Theorem 13.4] ${ }^{10}$ In fact, every super-real field is isomorphic to a field $K_{P}$ where $P$ is a non-maximal prime in $C(\Omega)$ for some compact space $\Omega$.

There is a substantial classification of prime ideals in algebras of continuous functions in [9]. We have noted that always $A_{P} \subset K_{P}^{\sharp}$. In fact, $A_{P}=K_{P}^{\sharp}$ if and only if $A_{P}$ is a valuation prime, in the sense that $A_{P}$ is a valuation algebra; see $[\mathbf{9}$, Proposition 4.32]. This is not always the case. Here is an easy preliminary remark: there are valuation primes that are not $z$-ideals, and there are prime $z$-ideals that are not valuation primes.

We now see that there is a further normability condition that would give a solution to Kaplansky's problem (in one direction).

THEOREM 2.7. Let $\Omega$ be a compact space. Suppose that there is a non-maximal, prime ideal $P$ in $C(\Omega)$ such that the algebra $K_{P}^{\sharp}$ of finite elements in the superreal field $K_{P}$ is normable. Then there is an algebra norm on $C(\Omega, \mathbb{C})$ that is not equivalent to the uniform norm.

This leads us to the question, which we shall consider below:

Let $K$ be an ordered field. When is the subalgebra $K^{\sharp}$ of finite elements of $K$ normable?

We remark that, by the Gel'fand-Mazur theorem in the form of $[\mathbf{6}$, Theorem $2.2 .42]$, the only real fields that are themselves normable are $\mathbb{R}$ and $\mathbb{C}$.

\footnotetext{
${ }^{10}$ These proofs use some elementary real analysis, and Rouché's theorem from complex analysis.
} 
2.7. Equivalence of compact spaces. It is natural to wonder if all infinite, compact spaces $\Omega$ are equivalent for Kaplansky's problem, in the sense that there is a discontinuous homomorphism for each $C(\Omega, \mathbb{C})$, where $\Omega$ is an infinite, compact space, whenever this is true for one particular compact space. The following theorem, from [8, Theorems 1.8 and 1.13] reduces this question to the consideration of the two spaces $\Omega=\beta \mathbb{N}$ and $\Omega=\mathbb{N} \cup\{\infty\}$ and the corresponding algebras $\ell^{\infty}(\mathbb{C})$ and $c_{0}(\mathbb{C})^{\sharp}$.

In the following, $J_{x}$ is regarded as an ideal in $C(\beta \mathbb{N}, \mathbb{C})$ for $x \in \beta \mathbb{N}$.

THEOREM 2.8. (i) Assume that there is a discontinuous homomorphism from $C(\Omega, \mathbb{C})$ into a Banach algebra for some compact space $\Omega$. Then there exists $x \in \mathbb{N}^{*}$ and a radical homomorphism $\theta$ from $c_{0}(\mathbb{C})$ with $\operatorname{ker} \theta \supset J_{x} \cap c_{0}(\mathbb{C})$, and so there is a discontinuous homomorphism from $c_{0}(\mathbb{C})$ into a Banach algebra.

(ii) Assume that there is a discontinuous homomorphism from $\ell^{\infty}(\mathbb{C})$ into a Banach algebra. Then there is a discontinuous homomorphism from $C(\Omega, \mathbb{C})$ into a Banach algebra for each infinite, compact space $\Omega$.

We shall reformulate the above theorem in the language of ultrafilters. Here $\mathcal{U}$ and $\mathcal{V}$ are free ultrafilters on $\mathbb{N}$.

The above theorem tells us the following: Assume that there exists $\mathcal{U}$ such that $\left(\mathbb{R}^{\omega} / \mathcal{U}\right)^{\circ}$ is semi-normable. Then there exists $\mathcal{V}$ such that $c_{0} / \mathcal{V}$ is semi-normable. This suggests the following questions:

(1) Assume that there exists $\mathcal{U}$ such that $c_{0} / \mathcal{U}$ is semi-normable. Does there exist $\mathcal{V}$ such that $\left(\mathbb{R}^{\omega} / \mathcal{V}\right)^{\circ}$ is semi-normable?

(2) Assume that $c_{0} / \mathcal{U}$ is semi-normable. Is $\left(\mathbb{R}^{\omega} / \mathcal{U}\right)^{\circ}$ semi-normable?

(3) Assume that $\left(\mathbb{R}^{\omega} / \mathcal{U}\right)^{\circ}$ is semi-normable. Is $c_{0} / \mathcal{U}$ semi-normable?

(4) Assume that $\left(\mathbb{R}^{\omega} / \mathcal{U}\right)^{\circ}$ is semi-normable. Is $\left(\mathbb{R}^{\omega} / \mathcal{U}\right)^{\circ}$ normable?

A positive answer to (1) would show that all infinite, compact spaces $\Omega$ are equivalent for Kaplansky's problem. Questions (2) and (3) are variants of Question (1) that seem to be of interest in their own right. A positive answer to Question (4) would cleanse the theory. We seem not to have an answer to any of these questions.

We shall see that, in the theory $\mathrm{ZFC}+\mathrm{CH}$, the four algebras $\left(\mathbb{R}^{\omega} / \mathcal{U}\right)^{\circ}$, $\left(\mathbb{R}^{\omega} / \mathcal{V}\right)^{\circ}, c_{0} / \mathcal{U}$, and $c_{0} / \mathcal{V}$ are all pairwise isomorphic (as real algebras) for any two free ultrafilters $\mathcal{U}$ and $\mathcal{V}$; this was first proved by Barry Johnson in [19]. However, it follows from a theorem of Dow [10, Corollary 2.3] that, if $\mathrm{CH}$ fails, there are two free ultrafilters $\mathcal{U}$ and $\mathcal{V}$ such that $\left(\mathbb{R}^{\omega} / \mathcal{U}\right)^{\circ}$ and $\left(\mathbb{R}^{\omega} / \mathcal{V}\right)^{\circ}$ are not isomorphic even as rings.

\section{Structure of ordered sets, groups, and fields}

We shall now recall the notions of $\alpha_{1}^{-}, \beta_{1}-$, and $\eta_{1}-$ structures on totally ordered sets, totally ordered groups, and ordered fields. These notions go back to Hausdorff around 1907. ${ }^{11}$ See $[\mathbf{9}$, Chapter 1].

\footnotetext{
${ }^{11}$ The subscript ' 1 ' is associated with the cardinal $\aleph_{1}$; there are similar notions for larger cardinals.
} 
3.1. Definitions and examples. Let $(S, \leq)$ be a totally ordered set. Then $S$ is an $\alpha_{1}-$ set if each non-empty subset of $S$ has a countable cofinal and coinitial subset, and an $\eta_{1}$-set if, for each countable subsets $S_{1}$ and $S_{2}$ of $S$ (including the case where either $S_{1}$ or $S_{2}$ is empty) with $S_{1} \ll S_{2}$, there exists an element $s \in S$ with $S_{1} \ll\{s\} \ll S_{2}$. For example, $(\mathbb{R}, \leq)$ is an $\alpha_{1}$-set, but not an $\eta_{1}$-set. Thus we think of $S$ as 'small' if it is an $\alpha_{1}$-set and 'large' if it is an $\eta_{1}$-set; clearly no totally ordered set is both an $\alpha_{1}$-set and an $\eta_{1}$-set. But an $\eta_{1}$-set can be 'nearly' an $\alpha_{1}$-set if it is a $\beta_{1}$-set: $S$ is a $\beta_{1}-$ set if it is the union of a chain of $\alpha_{1}$-subsets of $S$.

In fact there is 'exactly one' ordered set that is both a $\beta_{1}$-set and an $\eta_{1}$-set.

We denote by $\mathbf{Q}$ the set of all sequences $\left(\alpha_{\tau}: \tau<\omega_{1}\right)$ of length $\omega_{1}$, where $\alpha_{\tau} \in\{0,1\}\left(\tau<\omega_{1}\right)$, for which the set $\left\{\tau<\omega_{1}: \alpha_{\tau}=1\right\}$ is non-empty and has a largest member; this set $\mathbf{Q}$ is taken with the the lexicographic order $\preceq$. The following theorem is part of [9, Proposition 1.9 and Theorem 1.15].

THEOREM 3.1. The pair $(\mathbf{Q}, \preceq)$ is a $\beta_{1}-\eta_{1}$-set. Further, $|\mathbf{Q}|=w(\mathbf{Q})=\mathfrak{c}$ and $\operatorname{cof} \mathbf{Q}=\aleph_{1}$, and $(\mathbf{Q}, \preceq)$ is unique, in the sense that each $\beta_{1}-\eta_{1}$-set is orderisomorphic to $(\mathbf{Q}, \preceq)$.

The ordered set $\mathbf{Q}$ is called Sierpinski's set. This set is universal in the class of $\beta_{1}$-sets: every $\beta_{1}$-set is order-isomorphic to a subset of $\mathbf{Q}$.

Now take $(G,+, \leq)$ to be a totally ordered group. Then $G$ is an $\alpha_{1}$-group if $(G, \leq)$ is an $\alpha_{1}$-set and an $\eta_{1}-$ group if $(G, \leq)$ is an $\eta_{1}$-set. Further, $G$ is a $\beta_{1}$-group if it is the union of a chain of $\alpha_{1}$-subgroups. ${ }^{12}$ In fact there is 'exactly one' divisible group that is both a $\beta_{1}$-group and an $\eta_{1}$-set.

We define $\mathbf{G}$ to be $\mathfrak{F}_{(1)}(\mathbf{Q})$, in the notation of $\S 2.4$. The following theorem is part of [9, Propositions 1.43 and 1.44 and Theorem 1.48].

TheOREM 3.2. The triple $(\mathbf{G},+, \leq)$ is a divisible $\beta_{1}-\eta_{1}$-group. Further,

$$
|\mathbf{G}|=w(\mathbf{G})=\mathfrak{c} \quad \text { and } \quad \operatorname{cof} \mathbf{G}=\delta(\mathbf{G})=\aleph_{1},
$$

and $(\mathbf{G},+, \leq)$ is unique, in the sense that each divisible $\beta_{1}-\eta_{1}-$ group is isomorphic to $(\mathbf{G},+, \leq)$.

In particular, any two divisible $\eta_{1}$-groups of cardinality $\aleph_{1}$ are isomorphic. The group $\mathbf{G}$ is universal in the class of divisible $\beta_{1}$-groups: every divisible $\beta_{1}$-group is isomorphic to a subgroup of $\mathbf{G}[\mathbf{9}$, Theorem 1.46].

We finally step up further to consider fields.

Let $K=(K,+, \cdot, \leq)$ be an ordered field (still over $\mathbb{R})$. Then $K$ is an $\alpha_{1}$-field if $(K, \leq)$ is an $\alpha_{1}$-set and an $\eta_{1}$-field if $(K, \leq)$ is an $\eta_{1}$-set. Further, $K$ is a $\beta_{1}$-field if it is the union of a chain of $\alpha_{1}$-subfields. The subfields in the definition of a $\beta_{1}$-field must be fields over $\mathbb{R}$; maybe apparently weaker hypotheses are already sufficient to imply this.

EXAMPLE 3.3. Each hyper-real field is an $\eta_{1}$-field; this is a very classical fact from Gillman and Jerison [17, §13.8].

Now suppose that $\Omega$ is a compact space and that $P$ is a non-maximal prime ideal in $C(\Omega)$. Then the super-real field $K_{P}$ is an $\eta_{1}$-field if and only if $\delta\left(K_{P}\right) \geq \aleph_{1}$ [9, Theorem 4.28(ii)]. Thus super-real fields are 'usually' real-closed $\eta_{1}$-fields.

\footnotetext{
${ }^{12}$ Note that it is apparently not sufficient that $G$ be a union of a chain of $\alpha_{1}$-subsets.
} 
Let us consider the question whether the class, called super-real $\eta_{1}$ in [9], of super-real fields that are $\eta_{1}$-fields is actually the same as the class, hyper-real, of hyper-real fields, in the sense that each member of super-real $\eta_{1}$ is isomorphic as a field to a member of hyper-real. This seems to be a substantial question that we shall remark on below.

There is an example of a super-real field $K_{P}$ such that $P$ is a prime $z$-ideal in some $C(\Omega)$, but $K_{P}$ is not an $\eta_{1}$-field [9, Theorem 4.47]. This suggest consideration of the class $z$-ideal $\eta_{1}$ of super-real $\eta_{1}$-fields that are isomorphic to a field $K_{P}$, where $P$ is a prime $z$-ideal in some $C(\Omega)$. We do not know whether this class is the same as the class hyper-real. There are intermediate possibilities; see Question 12 , p. 342 , of $[\mathbf{9}]$.

In fact there is 'exactly one' real-closed ordered field that is both a $\beta_{1}$-field and an $\eta_{1}$-set. We now define this field, which is called $\mathbf{R}$, to be

$$
\mathbf{R}=\mathfrak{F}_{(1)}(\mathbf{G})=\mathfrak{F}_{(1)}\left(\mathfrak{F}_{(1)}(\mathbf{Q})\right) .
$$

The following theorem is part of [9, Theorem 2.23 and Corollary 2.33].

ThEOREM 3.4. The field $(\mathbf{R},+, \cdot, \leq)$ is a real-closed $\beta_{1}-\eta_{1}$-field, with value group G. Further,

$$
|\mathbf{R}|=w(\mathbf{R})=\mathfrak{c} \quad \text { and } \quad \operatorname{cof} \mathbf{R}=\delta(\mathbf{R})=\aleph_{1},
$$

and $(\mathbf{R},+, \cdot, \leq)$ is unique, in the sense that each real-closed $\beta_{1}-\eta_{1}$-field is isomorphic to $(\mathbf{R},+, \cdot, \leq)$ as an ordered field.

In particular, any two real-closed $\eta_{1}$-fields of cardinality $\aleph_{1}$ are isomorphic. The field $\mathbf{R}$ is universal in the class of $\beta_{1}$-fields: every $\beta_{1}$-field is isomorphic to a subfield of $\mathbf{R}$ [9, Theorem 2.30].

3.2. Positive results on normability. The original proofs in [5] and [12] evolved into the following theorem, taken from [6, Theorem 5.7.18]. The proof depends heavily on the construction of a 'framework map', described in [6, $\S 1.7]$, and is still quite substantial, involving transfinite induction and algebraic manipulations. The theorem holds in the theory ZFC.

THEOREM 3.5. The algebra $\mathbf{R}^{\sharp}$ of finite elements in the real-closed $\beta_{1}-\eta_{1}$-field $\mathbf{R}$ is normable.

We immediately obtain the following consequence from the above remarks.

THEOREM 3.6. Let $K$ be a $\beta_{1}$-field. Then $K^{\sharp}$ is normable. In particular, let $\Omega$ be a compact space, and suppose that $P$ is a non-maximal, prime ideal in $C(\Omega)$ for which $K_{P}$ is a $\beta_{1}$-field. Then there is an embedding of $C(\Omega) / P$ into a commutative Banach algebra.

Recall that, for each infinite compact space $\Omega$, the algebra $C(\Omega, \mathbb{C})$ contains a maximal ideal $M_{x}$ and a prime ideal $P$ with $J_{x} \subset P \subsetneq M_{x}$ and $\left|M_{x} / P\right|=\mathfrak{c}$. With $\mathrm{CH},\left|M_{x} / P\right|=\aleph_{1}$, and so $K_{P}$ is a $\beta_{1}$-field. This recovers Theorem 1.8.

In fact, using remarks in $\S 2.6$, we see that the following slightly stronger theorem holds [6, Theorem 5.7.13(ii)]. 
TheOREM 3.7. Assume that there is a free ultrafilter $\mathcal{U}$ on $\mathbb{N}$ such that the ultrapower $\mathbb{R}^{\omega} / \mathcal{U}$ is a $\beta_{1}$-field. Then there is a a discontinuous homomorphism from $C(\Omega, \mathbb{C})$ into a Banach algebra for each infinite compact space $\Omega$.

Clearly one would obtain a result without appealing to $\mathrm{CH}$ if one could find a free ultrafilter $\mathcal{U}$ on $\mathbb{N}$ such that the ultrapower $\mathbb{R}^{\omega} / \mathcal{U}$ is a $\beta_{1}$-field. However this is not easy. First, whenever $\mathrm{CH}$ fails, there is always some free ultrafilter $\mathcal{U}$ on $\mathbb{N}$ such that the ultrapower $\mathbb{R}^{\omega} / \mathcal{U}$ is not a $\beta_{1}$-field. Further, we have the following result from [9, Corollary 6.28]; here, MA is 'Martin's axiom'. Of course, CH implies MA.

THEOREM 3.8. (MA $+\neg \mathrm{CH})$ For each free ultrafilter $\mathcal{U}$ on $\mathbb{N}$, the ordered set $\left(\mathbb{R}^{\omega} / \mathcal{U}, \leq\right)$ is not a $\beta_{1}-$ set.

Thus, to follow the above route, one must work in a theory where MA does not hold. This has been carried out by Woodin in [30] in a special case.

THEOREM 3.9. Assume that ZFC is consistent. Then there is a model of the theory $\mathrm{ZFC}+\neg \mathrm{MA}$ in which there is a free ultrafilter $\mathcal{U}$ on $\mathbb{N}$ such that the ultrapower $\mathbb{R}^{\omega} / \mathcal{U}$ is a $\beta_{1}$-field, and hence such that there is a discontinuous homomorphism from $C(\Omega, \mathbb{C})$ into a Banach algebra for each infinite compact space $\Omega$.

This shows that $\neg \mathrm{NDH}$ is not equivalent to $\mathrm{CH}$ in $\mathrm{ZFC}$.

Hugh obtained his model by adding $\aleph_{2}$ Cohen reals to a model of ZFC $+\mathrm{CH}$. In particular, in this model $\mathfrak{c}=\aleph_{2}$. It seems to remain open whether one can obtain such a model with $\mathfrak{c}>\aleph_{2}$. It also remains open whether, given a model of ZFC, there is a model of $\mathrm{ZFC}+\mathrm{MA}+\neg \mathrm{NDH}$; for a remark on this involving the proper forcing axiom, see below.

3.3. Other algebras. We have shown that, with $\mathrm{CH}$, the integral domains of the form $C(\Omega, \mathbb{C}) / P$ are normable when they have cardinality $\mathfrak{c}$. This result can be generalized; the following theorem is $[\mathbf{9}$, Theorem 5.25], extending [6, Theorem $5.7 .10]$.

THEOREM 3.10. Let $A$ be a non-unital, complex integral domain with $|A|=\mathfrak{c}$. Then there is a free ultrafilter $\mathcal{U}$ on $\mathbb{N}$ such that there is an embedding of $A$ into $c_{0}(\mathbb{C}) / \mathcal{U}$.

Corollary 3.11. (CH) Let A be a non-unital, complex integral domain with $|A|=\mathfrak{c}$. Then $A$ is normable.

The above result suggests that, with $\mathrm{CH}$, there is a discontinuous homomorphism into a Banach algebra from each infinite-dimensional, commutative Banach algebra. Towards this, we have the following theorem [6, Theorem 5.7.32].

THEOREM 3.12. ( $\mathrm{CH})$ Let $A$ be an infinite-dimensional, commutative Banach algebra satisfying at least one of the following conditions:

(i) there is a non-maximal, prime ideal $P$ in $A$ with $|A / P|=\mathfrak{c}$;

(ii) there are infinitely many distinct characters on $A$;

(iii) for each $a \in A$, the set $\left\{a^{n}: n \in \mathbb{N}\right\}$ is linearly dependent;

(iv) the linear subspace spanned by $\{a b: a, b \in A\}$ has infinite codimension in the algebra $A$.

Then there is discontinuous homomorphism from $A$ into some Banach algebra. 
I have not been able to find an infinite-dimensional, commutative Banach algebra that does not satisfy at least one of the above four conditions, and it is conceivable that every infinite-dimensional, commutative Banach algebra satisfies at least one of them, in which case our question would be solved. However, so far, the question whether there is a discontinuous homomorphism into a Banach algebra from each infinite-dimensional, commutative Banach algebra remains open.

\section{Related questions with $\mathrm{CH}$}

There is a variety of related questions when we do assume $\mathrm{CH}$, and we mention some of these here.

4.1. The range of radical homomorphisms. In Theorem 3.5, we stated that the algebra $\mathbf{R}^{\sharp}$ is normable. This says that there is an embedding of $\mathbf{R}^{\circ}$ into a commutative, radical Banach algebra. One could ask: Into which commutative, radical Banach algebras can we embed $\mathbf{R}^{\circ}$ ?

We already know from Allan's theorem, Theorem 2.4, that we can embed the maximal ideal $\mathfrak{F}^{\circ}$ of $\mathfrak{F}$ into a commutative, radical Banach algebra $R$ if and only if $R$ contains an element of finite closed descent. It is remarkable that the latter class of commutative, radical Banach algebras is exactly the class into which we can embed the 'much bigger' algebra $\mathbf{R}^{\circ}$.

There are further characterizations of these algebras. We use the notation $\mathbb{R}^{+\bullet}=\{x \in \mathbb{R}: x>0\}$ and $\mathbb{Q}^{+\bullet}=\mathbb{R}^{+\bullet} \cap \mathbb{Q}$, and take $A$ to be an algebra. A real semigroup, respectively, rational semigroup, in $A$ is a morphism $\psi: \mathbb{R}^{+\bullet} \rightarrow(A, \cdot)$, respectively, $\psi: \mathbb{Q}^{+\bullet} \rightarrow(A, \cdot)$, and these semigroups are non-zero if their range does not contain 0 .

The following theorem is due to Esterle [15]; see [6, Theorem 5.7.28].

Theorem 4.1. Let $R$ be a commutative, radical Banach algebra. Then the following conditions on $R$ are equivalent:

(a) there is an embedding of $\mathfrak{F}^{\circ}$ into $R$;

(b) there is an embedding of $\mathbf{R}^{\circ}$ into $R$;

(c) $R$ contains an element of finite closed descent;

(d) $R$ contains a real semigroup;

(e) $R$ contains a rational semigroup;

(f) there is a sequence $\left(a_{n}\right)$ in $R \backslash\{0\}$ such that $a_{n} \in a_{n+1}^{2} R(n \in \mathbb{N})$.

There are many further examples of commutative, radical Banach algebras satisfying the above conditions in $[\mathbf{6}]$. Here is a particularly easy one. Let $A(\overline{\mathbb{D}})$ be the disc algebra of all continuous functions on the the closed unit disc $\overline{\mathbb{D}}$ that are analytic on the open unit disc $\mathbb{D}$. Then $A(\overline{\mathbb{D}})$ is a uniformly closed subalgebra of $C(\overline{\mathbb{D}})$. Let $M=\{f \in A(\overline{\mathbb{D}}): f(1)=0\}$, so that $M$ is a maximal ideal in $A(\overline{\mathbb{D}})$. Define

$$
f_{0}(\zeta)=\exp \left(\frac{\zeta+1}{\zeta-1}\right) \quad(\zeta \in \overline{\mathbb{D}}),
$$

with $f_{0}(1)=0$. Then $f_{0} M$ is a closed ideal in $M$, and $M / f_{0} M$ is a commutative, radical Banach algebra that satisfies the equivalent conditions of Theorem 4.1. 
4.2. The normability of quotients. We have shown that, with $\mathrm{CH}$, for each non-maximal, prime ideal $P$ in an algebra $C(\Omega)$ such that $|C(\Omega) / P|=\mathfrak{c}$, the quotient algebra $C(\Omega) / P$ is normable. The natural question that arises is: Let $I$ be a non-maximal ideal in $C(\Omega)$. When is $C(\Omega) / I$ normable?

An easy extension of the main theorem shows that, with $\mathrm{CH}$, a quotient $C(\Omega) / I$ is normable whenever $|C(\Omega) / I|=\mathfrak{c}$ and the ideal $I$ is a finite intersection of prime ideals. In [6], it was said to be a 'natural conjecture' that the continuity ideal $\mathcal{I}(\theta)$ of a discontinuous homomorphism $\theta$ from an algebra $C(\Omega)$ into a Banach algebra is always a finite intersection of prime ideals. This natural conjecture was proved by Esterle in [12] for various compact spaces $\Omega$, including $\beta \mathbb{N}$ and $\mathbb{N}^{*}$; see $[\mathbf{6}$, Theorem 5.4.35]. However this conjecture turned out to be false, as shown by work of Hung Le Pham.

Here is a weaker result than the conjecture. First we give a definition from $[\mathbf{2 1}$, Definition 3.1] and [23, Definition 3.2].

Definition 4.2. Let $A$ be a commutative algebra. A family $\left\{P_{i}: i \in S\right\}$ of prime ideals in $A$ is pseudo-finite if $a \in P_{i}$ for all save finitely many $i \in S$ whenever $a \in P_{i}$ for some $i \in S$. A family $\mathfrak{P}$ of prime ideals in $A$ is relatively compact if every sequence of prime ideals in $\mathfrak{P}$ contains a pseudo-finite subsequence.

There is a sense in which there is a topology on the set of prime ideals in $A$ that makes a relatively compact family according to the above definition into a relatively compact set with respect to this topology. Pham proved the following theorem in $[\mathbf{2 3}]$.

THEOREM 4.3. Let $\Omega$ be a locally compact, non-compact space, and let $\theta$ be a homomorphism from $C_{0}(\Omega)$ into a commutative, radical Banach algebra. Then $\operatorname{ker} \theta=\mathcal{I}(\theta)$ is the intersection of a relatively compact family of non-modular prime ideals in $C_{0}(\Omega)$.

The most interesting aspect of the above theorem is that the converse is 'almost true', and so we are close to characterizing the ideals $I$ in $C_{0}(\Omega)$ that are the kernels of radical homomorphisms from $C_{0}(\Omega)$. The next theorem is taken from [23, Theorems 6.7 and 6.8].

THEOREM 4.4. (CH) Let $\Omega$ be a locally compact, non-compact space, and let $I$ be an ideal in $C_{0}(\Omega)$ with $\left|C_{0}(\Omega) / I\right|=\mathfrak{c}$. Suppose that $I$ is the intersection of a relatively compact family $\mathfrak{P}$ of non-modular prime ideals in $C_{0}(\Omega)$ satisfying the additional condition that every chain in the closure of $\mathfrak{P}$ is countable. Then there is a homomorphism with kernel I from $C_{0}(\Omega)$ into a commutative, radical Banach algebra, and so $C_{0}(\Omega) / I$ is normable.

The closure of $\mathfrak{P}$ in the above theorem is the set of all the unions of pseudofinite sequences in $\mathfrak{P}$; the latter are necessarily non-modular, prime ideals in $C_{0}(\Omega)$. Every chain in the closure of $\mathfrak{P}$ is well-ordered by inclusion; if $\mathfrak{P}$ is countable, then every chain in its closure is indeed countable. It is possible that the 'additional condition' in the above theorem is redundant.

The point of Theorem 4.4 is that there are many uncountable, locally compact, metrizable spaces $\Omega$, including the real line $\mathbb{R}$ itself, and corresponding ideals $I$ that satisfy the conditions in that theorem, without being finite intersections of primes, and so there are ideals $I$ in $C_{0}(\Omega)$ such that $C_{0}(\Omega) / I$ is normable, but $I$ is not a 
finite intersection of prime ideals. Indeed, Pham proves in $[\mathbf{2 2}]$ that, with $\mathrm{CH}$, there exists a radical homomorphism from $C_{0}(\mathbb{R})$ whose kernel is not the intersection of even countably many prime ideals.

4.3. $C^{*}$-algebras. As we remarked there are classical positive results about the automatic continuity of arbitrary homomorphisms from (non-commutative) Banach algebras into Banach algebras $[6]$. The case when the domain is a $C^{*}$-algebra is particularly interesting. For example, let $H$ be a Hilbert space. Then each homomorphism from $\mathcal{B}(H)$, the $C^{*}$-algebra of all bounded operators on $H$, into a Banach algebra is automatically continuous [6, Corollary 5.4.13]; more general results are due to Johnson in [18].

The analogue of the theorem of Bade and Curtis giving the structure of an arbitrary homomorphism $\theta$ from a $C^{*}$-algebra $A$ into a Banach algebra $B$ such that $\overline{\theta(A)}=B$ is mainly due to Sinclair [24]; see [6, Theorem 5.4.40].

Discontinuous homomorphisms from general $C^{*}$-algebras are constructed in $[\mathbf{6}$, Theorem 5.7.35] as follows.

TheOrem 4.5. (CH) Let $A$ be a Banach algebra such that $A$ has infinitely many non-equivalent simple representations of dimension $k^{13}$ for some $k \in \mathbb{N}$. Then there is a discontinuous homomorphism from $A$ into some Banach algebra.

This suggested the following question, raised as Question 5.4.A in [6]: Let $A$ be $a C^{*}$-algebra, such that, for each $k \in \mathbb{N}$, there are only finitely many non-equivalent simple representations of $A$ of dimension $k$. Does it follow that each homomorphism from A into a Banach algebra is automatically continuous? If true, such a result would characterize the $C^{*}$-algebras such that all homomorphisms from the algebra are continuous and generalize all known theorems on the automatic continuity of homomorphisms from $C^{*}$-algebras. $5.4 .27]$

The following pleasing result was proved by Esterle in $[\mathbf{1 4}]$; see $[\mathbf{6}$, Theorem

THEOREM 4.6. Let $\Omega$ be a non-empty, compact space. Then every epimorphism from $C(\Omega)$ onto a Banach algebra is automatically continuous.

Of course, this suggested the following question, raised as Question 5.4.E in [6]: Is every epimorphism from a $C^{*}$-algebra onto a Banach algebra automatically continuous?

I regret to report that it seems that no conclusive progress has been made on either of the above two questions. For the best partial results, see [11].

\section{The independence of NDH}

We now come to consideration of the fact that NDH and the solution to Kaplansky's problem is independent of ZFC; we shall discuss the early work of Woodin. It may be of interest to record some history.

\footnotetext{
${ }^{13} \mathrm{~A}$ representation of dimension $k$ of an algebra $A$ is a homomorphism from $A$ into the algebra of $k \times k$ matrices acting on $\mathbb{C}^{k}$; the representation is simple if the only subspaces of $\mathbb{C}^{k}$ that are invariant under all images of elements of $A$ are trivial.
} 
5.1. Historical remarks. The paper [5] was mainly worked out in the year 1973-74, whilst I was at UCLA; I thank Phil. Curtis for inviting me to UCLA and for much support. I gave lectures on this at the inaugural conference on Banach algebras in July, 1974. The work was mostly written in the year 1974-75.

Let $P$ be a non-maximal, prime ideal in $C(\Omega)$ with $\left|A_{P}\right|=\mathfrak{c}$. For a long time, I had a construction of a non-zero homomorphism from a 'large' subalgebra, $B$, of $A_{P}$ into a commutative, radical Banach algebra, but I could not see that $B$ was the whole of $A_{P}$. Suddenly, I realised that, whilst maybe $B \neq A_{P}$, it was the case that, with $\mathrm{CH}, B$ was isomorphic to $A_{P}$, and that this was sufficient to obtain Theorem 1.8. The paper [5] was submitted in May, 1976.

I then thought how one could remove ' $(\mathrm{CH})$ ' from the theorem, but could not do this; with some trepidation I wrote in June 1976 to Professor Robert Solovay at Berkeley, and asked for his help in this. (Young colleagues might like to know that one wrote by hand on paper in those distant days; but we did have airmail.) This letter eventually reached Solovay at Caltech, where he was on leave.

The next part of the story is based on information from Frederick Dashiell, then a Bateman Research Instructor at Caltech; I am grateful to Fred for this.

Hugh Woodin, then a junior at Caltech, approached Fred in January 1976 for a topic for his senior thesis. Fred suggested that Hugh organize what was known about Kaplansky's problem up to that point, and explain the heart of the open question on homomorphisms from $C(\beta \mathbb{N}, \mathbb{C})$. Hugh ignored the survey part of the suggestion, and immediately started trying to construct a model of NDH; he was learning about forcing at that time, and discussed the question with Solovay, the leading expert on forcing. Hugh produced a type-written document [28] that I have in 'Fall 1976'; by that time Hugh had seen the preprint of [5]. It seems that this document was not submitted as a senior thesis, and that it has not been published, but results from it are contained in Hugh's thesis [29] and in [8]. The paper [28] seems to be Hugh's first contribution to mathematics.

In fact, Hugh proved in $[\mathbf{2 8}, \S 5]$ that it is consistent with ZFC that there is an ultrafilter $\mathcal{U}$ such that $\ell^{\infty} / \mathcal{U}$ does not admit a non-zero algebra semi-norm, and, in [28, §6], he gave a set-theoretic condition, now 'Woodin's condition', which, if satisfied, implies NDH. Subsequently Solovay showed that this condition was consistent with ZFC, and lectured on this on 26 October 1976; this argument was never published because Hugh himself soon gave a shorter proof of the same result. I am impressed that, as an undergraduate, Hugh could confidently use such sentences as: 'Let $\mathfrak{M}$ be a countable standard model of $\mathrm{ZFC}+\mathrm{CH}$, and let $\mathfrak{N}$ be the extension obtained by adding $\omega_{2}$ Cohen reals.'

In the work [28], Hugh also discusses 'weak semi-normability'; some of these remarks reappear in [8].

After some time Solovay kindly replied to my letter, saying that 'Woodin' had shown that there are models of set theory in which NDH is true. I wrote to the person that I took to be 'Professor Woodin' at Caltech to ask about this, and received $[\mathbf{2 8}]$ in response.

5.2. Woodin's condition. I wish to recall the proof of Woodin's condition; it is taken from [8, Chapter 3].

Recall first that the divisibility order $\ll$ and the strong Fréchet order $\ll_{\mathcal{F}}$ were defined in Examples 2.1. 
Let $g \in \mathbb{N}^{\mathbb{N}}$ with $1 \ll_{\mathcal{F}} g$, where 1 now denotes the sequence that is constantly equal to 1 . Then

$$
\langle g\rangle=\left\{f \in \mathbb{N}^{\mathbb{N}}: 1 \ll_{\mathcal{F}} f \ll_{\mathcal{F}} g\right\},
$$

so that $\left(\langle g\rangle, \ll_{\mathcal{F}}\right)$ is a partially ordered set. We regard $\left(\langle g\rangle_{\mathcal{U}},<_{\mathcal{U}}\right)$ as a totally ordered subset of the ultrapower $\left(\mathbb{R}^{\omega} / \mathcal{U},<\mathcal{U}\right)$.

ThEOREM 5.1. Assume that there is a free ultrafilter $\mathcal{U}$ on $N$ such that $c_{0} / \mathcal{U}$ is semi-normable. Then there is an unbounded, monotonically increasing function $g \in \mathbb{N}^{\mathbb{N}}$ and an isotonic map from $\left(\langle g\rangle_{\mathcal{U}},<_{\mathcal{U}}\right)$ into $\left(\mathbb{N}^{\mathbb{N}},<_{\mathcal{F}}\right)$.

ProOF. There is a commutative, radical Banach algebra $(R,\|\cdot\|)$ and a nonzero homomorphism $\theta: c_{0} / \mathcal{U} \rightarrow R$. Choose $a \in c_{0} / \mathcal{U}$ with $\theta(a) \neq 0$; we may suppose that $a>0$. Then choose a sequence $\alpha \in c_{0}$ such that $[\alpha]_{\mathcal{U}}=a$; we may suppose that $0<\alpha_{n}<1(n \in \mathbb{N})$. Finally, choose an unbounded, monotonically increasing function $g \in \mathbb{N}^{\mathbb{N}}$ such that

$$
\frac{1}{g(n)^{2}} \log \left(\frac{1}{\alpha_{n}}\right) \rightarrow \infty \quad \text { as } \quad n \rightarrow \infty .
$$
position

We shall now construct an isotonic map $\pi:\left(\langle g\rangle_{\mathcal{U}},<_{\mathcal{U}}\right) \rightarrow\left(\mathbb{N}^{\mathbb{N}},<_{\mathcal{F}}\right)$ as a com-

$$
\left(\langle g\rangle_{\mathcal{U}},<\mathcal{U}\right) \stackrel{\nu}{\rightarrow}\left(c_{0} / \mathcal{U}, \ll\right) \stackrel{\theta}{\rightarrow}(R \backslash \operatorname{nil} R, \ll) \stackrel{\tau}{\rightarrow}\left(\mathbb{N}^{\mathbb{N}},<\mathcal{F}\right) .
$$

The map $\theta: c_{0} / \mathcal{U} \rightarrow R$ is given, and clearly this map is isotonic; the maps $\nu$ and $\tau$ will be anti-isotonic.

Take $f \in\langle g\rangle$, and set

$$
\alpha^{f / g^{2}}=\left(\alpha_{n}^{f(n) / g(n)^{2}}\right) .
$$

It follows from (5.1) that $\alpha^{f / g^{2}} \in c_{0} \backslash\{0\}$. For $[f] \in\langle g\rangle_{\mathcal{U}}$, set

$$
\nu([f])=\left[\alpha^{f / g^{2}}\right] \in c_{0} / \mathcal{U}
$$

the map $\nu:\langle g\rangle_{\mathcal{U}} \rightarrow c_{0} / \mathcal{U}$ is well-defined. Suppose that $\left[f_{1}\right]<\mathcal{U}\left[f_{2}\right]$ in $\langle g\rangle_{\mathcal{U}}$; we may suppose that $1 \leq f_{1}(n)<f_{2}(n) \leq g(n)(n \in \mathbb{N})$. Set

$$
\beta=\left(\alpha_{n}^{\left.\left(f_{2}(n)-f_{1}(n)\right) / g(n)^{2}\right)}\right. \text {. }
$$

Again by (5.1), we have $\beta \in c_{0} \backslash\{0\}$, and clearly $\nu\left(\left[f_{1}\right]\right)[\beta]=\nu\left(\left[f_{2}\right]\right)$, so that $\nu\left(\left[f_{2}\right]\right) \ll \nu\left(\left[f_{1}\right]\right)$ in $c_{0} / \mathcal{U}$. Thus $\nu:\left(\langle g\rangle_{\mathcal{U}},<\mathcal{U}\right) \rightarrow\left(c_{0} / \mathcal{U}, \ll\right)$ is anti-isotonic.

We next claim that the range of $\theta \circ \nu$ is contained in $R \backslash$ nil $R$. Indeed, take $f \in\langle g\rangle$ and $k \in \mathbb{N}$, and set

$$
\gamma=\left(\alpha_{n}^{k f(n) / g(n)^{2}}\right)
$$

Then $\gamma \in c_{0}$ and $\nu([f])^{k}[\gamma]=a$, and so $(\theta \circ \nu)([f])^{k} \neq 0$, giving the claim.

For $x \in R \backslash$ nil $R$, define

$$
\tau(x)(n)=\min \left\{k \in \mathbb{N}: k \geq 1 /\left\|x^{n}\right\|\right\} \quad(n \in \mathbb{N}),
$$

so that $\tau(x) \in \mathbb{N}^{\mathbb{N}}$. Take $x, y \in R \backslash$ nil $R$ with $x \ll y$, say $x=y z$, where $z \in R$. Then $z \notin$ nil $R$, and

$$
\left\|x^{n}\right\|^{-1} \geq\left\|y^{n}\right\|^{-1}\left\|z^{n}\right\|^{-1} \quad(n \in \mathbb{N}) .
$$

Since $R$ is a radical algebra, $\left\|z^{n}\right\|^{-1} \rightarrow \infty$ as $n \rightarrow \infty$, and so $\tau(x)(n)>\tau(y)(n)$ eventually. Thus $\tau(y)<_{\mathcal{F}} \tau(x)$, and so $\tau:(R \backslash$ nil $R, \ll) \rightarrow\left(\mathbb{N}^{\mathbb{N}},<_{\mathcal{F}}\right)$ is anti-isotonic. 
Set $\pi=\tau \circ \theta \circ \nu:\left(\langle g\rangle_{\mathcal{U}},<_{\mathcal{U}}\right) \rightarrow\left(\mathbb{N}^{\mathbb{N}},<_{\mathcal{F}}\right)$. Then $\pi$ is an isotonic map, as required.

We write $Z: n \mapsto n$, so that $Z \in \mathbb{N}^{\mathbb{N}}$. The conclusion of the following theorem is Woodin's condition from [28].

THEOREM 5.2. Assume that there is a non-empty, compact space $\Omega$ and a discontinuous homomorphism from $C(\Omega, \mathbb{C})$ into a Banach algebra. Then there is a free ultrafilter $\mathcal{V}$ on $\mathbb{N}$ and an isotonic map from $\left(\langle Z\rangle_{\mathcal{V}},<_{\mathcal{V}}\right)$ into $\left(\mathbb{N}^{\mathbb{N}},<_{\mathcal{F}}\right)$.

Proof. Let $g$ and $\mathcal{U}$ be as specified in Theorem 5.1, and then define the set $\sigma_{n}$ to be $g^{-1}(\{n\})$ for $n \in \mathbb{N}$, so that each $\sigma_{n}$ is finite and $\left\{\sigma_{n}: n \in \mathbb{N}\right\}$ is a partition of $\mathbb{N}$. Define

$$
\mathcal{V}=\left\{\sigma \subset \mathbb{N}: \bigcup\left\{\sigma_{n}: n \in \sigma\right\} \in \mathcal{U}\right\} .
$$

Certainly $\mathcal{V}$ is a filter on $\mathbb{N}$. If $\sigma \subset \mathbb{N}$, then either $\sigma \in \mathcal{V}$ or $\mathbb{N} \backslash \sigma \in \mathbb{N}$, and so $\mathcal{V}$ is an ultrafilter on $\mathbb{N}$. Indeed $\mathcal{V}$ is a free ultrafilter on $\mathbb{N}$.

Take $f \in\langle Z\rangle$. Then $(f \circ g)(n) \rightarrow \infty$ and $g(n)-(f \circ g)(n)$ as $n \rightarrow \infty$, and so $f \circ g \in\langle g\rangle$. The map

$$
[f]_{\mathcal{V}} \mapsto[f \circ g]_{\mathcal{U}}, \quad\left(\langle Z\rangle_{\mathcal{V}},<\mathcal{V}\right) \rightarrow\left(\langle g\rangle_{\mathcal{U}},<_{\mathcal{U}}\right)
$$

is well-defined and isotonic. Thus the result follows from Theorem 2.8(i) and Theorem 5.1

The force of the above theorem is, of course, that in the case where (given a model of ZFC) there is a model of ZFC in which there is no isotonic map from $\left(\langle Z\rangle_{\mathcal{V}},<_{\mathcal{V}}\right)$ into $\left(\mathbb{N}^{\mathbb{N}},<_{\mathcal{F}}\right)$, it will follow that $\mathrm{ZFC}+\mathrm{NDH}$ is relatively consistent with ZFC. The construction of such a model was an achievement of Woodin; the construction is given in Chapter 6 of [8], where the theory of gaps in totally ordered sets is developed, and in Chapter 8 of [8], where we explain 'iterated forcing', following the seminal work of Solovay and Tennenbaum [26]. Explicitly we have the following theorem of Woodin [8, Theorem 8.25].

THEOREM 5.3. Assume that $\mathfrak{M}$ is a model such that $\mathfrak{M} \models \mathrm{ZFC}$. Then there is a model $\mathfrak{N}$ extending $\mathfrak{M}$ such that $\mathfrak{N} \models \mathrm{ZFC}+\mathrm{MA}+\mathrm{NDH}$.

The proper forcing axiom PFA is a widely-used strengthening of Martin's axiom, MA. Stevo Todorčević proved in his book [27] (see Theorem 8.8 and some remarks on page 87) that Woodin's condition follows from PFA. This means, first of all, that $\mathrm{NDH}$ is a theorem of $\mathrm{ZFC}+\mathrm{PFA}$. It also explains why it may be difficult to obtain models of $\mathrm{ZFC}+\mathrm{MA}+\neg \mathrm{NDH}$ since these cannot be models of PFA.

We conclude this section with the following theorem.

THEOREM 5.4. Assume that ZFC is consistent. Then $\mathrm{NDH}$ is independent from ZFC.

\section{Norming larger fields and super-real fields}

In $\S 2.6$, we raised the question: Let $K$ be an ordered field. When is the subalgebra $K^{\sharp}$ of finite elements of $K$ normable? In Theorem 3.5, we stated that this is true for the specific ordered field $\mathbf{R}$, and this implies that, with $\mathrm{CH}, K^{\sharp}$ is normable for each ordered field $K$ with $|K|=\mathfrak{c}$. Now we investigate whether this is true for some larger fields than $\mathbf{R}$. 
6.1. A theorem of Esterle. First we give a theorem of Jean Esterle [12] that gives an upper bound to the cardinality of fields $K$ such that $K^{\sharp}$ is normable; see [9, Theorem 5.3].

THEOREM 6.1. Let $K$ be an ordered field such that $K^{\sharp}$ is normable. Then $\left|\Gamma_{K}\right| \leq \mathfrak{c}$, and so $|K| \leq 2^{\mathfrak{c}}$.

Proof. The norm on $K^{\sharp}$ is denoted by $\|\cdot\|$.

First take $a, b \in K^{\circ} \backslash\{0\}$ with $v(a)>v(b)$, where $v$ is the archimedean valuation, so that $a=b c$ for some $c \in K^{\circ}$. Then $0<\left\|a^{n}\right\| \leq\left\|b^{n}\right\|\left\|c^{n}\right\|(n \in \mathbb{N})$. Since $\lim _{n \rightarrow \infty}\left\|c^{n}\right\|^{1 / n}=0$, it follows that $\left\|a^{n}\right\|<\left\|b^{n}\right\|$ eventually. The map

$$
\psi: a \mapsto\left(\left\|a^{n}\right\|\right), \quad K^{\circ} \backslash\{0\} \rightarrow \mathbb{R}^{\mathbb{N}},
$$

induces an injection from $\Gamma_{K}$ into $\mathbb{R}^{\mathbb{N}}$. Thus $\left|\Gamma_{K}\right| \leq\left|\mathbb{R}^{\mathbb{N}}\right|=\mathfrak{c}$.

Now assume GCH. Then we know whether $K^{\sharp}$ is normable for every ordered field $K$ except for fields that satisfy the following combination of cardinalities:

$$
|K|=\aleph_{2} \quad \text { and } \quad\left|\Gamma_{K}\right|=\aleph_{1} .
$$

The first question is whether there are any ordered fields satisfying $(*)$, and, if so, whether any fall into 'nice' classes such as the classes of ultrapowers, of hyperreal fields, or of super-real fields. Note that, with $\mathrm{CH}$, the ordered field $\mathbf{R}$ is an ultrapower, but that, in the theory $\mathrm{MA}+\neg \mathrm{CH}$, by a result related to Theorem $3.8, \mathbf{R}$ is not even order-isomorphic to a super-real field [9, Corollary 6.26].

It is shown fairly easily in $[\mathbf{9}$, Theorem 7.2$]$ that, with GCH, there is indeed a super-real field $K_{P}$, with $P$ a prime $z$-ideal, that satisfies $(*)$, and we can find a hyper-real field that satisfies $(*)$ if we are allowed the combinatorial principle 'diamond' $(\diamond)$, a principle that is consistent with ZFC. Is there an ultrapower that satisfies $(*)$ ? In [9, Theorem 7.18], it is shown that it cannot be proved in ZFC that there is not an ultrapower satisfying $(*)$.

The second question is whether $K^{\sharp}$ is normable for fields in this class. The first obvious conjecture is the following.

Conjecture 6.2 . Let $K$ be an ordered field such that $\Gamma_{K}$ is a $\beta_{1}$-group (so that $\left|\Gamma_{K}\right|=\aleph_{1}$ with $\left.\mathrm{CH}\right)$. Then $K^{\sharp}$ is normable.

A proof of the following weaker conjecture would still amount to an attractive strengthening of Theorem 3.6.

Conjecture 6.3. Let $K$ be an ordered field such that $w(K)=\aleph_{1}$. Then $K^{\sharp}$ is normable.

6.2. The field $\widehat{\mathbf{R}}$. Recall that, in $\S 3.1$, we defined $\mathbf{R}$ to be $\mathfrak{F}_{(1)}(\mathbf{G})$. We now define $\widehat{\mathbf{R}}$ to be the following 'much bigger' field than $\mathbf{R}$ :

$$
\widehat{\mathbf{R}}=\mathfrak{F}(\mathbf{G})=\mathfrak{F}\left(\mathfrak{F}_{(1)}(\mathbf{Q})\right) .
$$

The following theorem is part of [ $\mathbf{9}$, Theorem 2.23].

THeOREM 6.4. The field $(\widehat{\mathbf{R}},+, \cdot, \leq)$ is a real-closed $\eta_{1}$-field with value group G. Further,

$$
|\widehat{\mathbf{R}}|=w(\widehat{\mathbf{R}})=2^{\aleph_{1}} \quad \text { and } \quad \operatorname{cof} \widehat{\mathbf{R}}=\delta(\widehat{\mathbf{R}})=\aleph_{1} .
$$


The field $\widehat{\mathbf{R}}$ is universal in the class of fields $K$ such that $\Gamma_{K}$ is a $\beta_{1}$-group: every such field is isomorphic to a subfield of $\widehat{\mathbf{R}}[\mathbf{9}$, Theorem 2.24]. Since $|\mathbf{R}|=\mathfrak{c}$ and $|\widehat{\mathbf{R}}|=2^{\aleph_{1}}$, it is possible that $|\mathbf{R}|=|\widehat{\mathbf{R}}|$, but the two fields are different, in the sense that they are not even order-isomorphic [9, Theorem 2.23(vi)]. In particular, $\widehat{\mathbf{R}}$ is not a $\beta_{1}$-field.

We see that, with GCH, $|\widehat{\mathbf{R}}|=\aleph_{2}$ and $\left|\Gamma_{\widehat{\mathbf{R}}}\right|=\aleph_{1}$, and so $\widehat{\mathbf{R}}$ satisfies $\left({ }^{*}\right)$, above.

In fact, the field $\widehat{\mathbf{R}}$ is a test case for Conjecture 6.2: the algebra $K^{\sharp}$ is normable for each ordered field $K$ such that $\Gamma_{K}$ is a $\beta_{1}$-group if and only if $\widehat{\mathbf{R}}^{\sharp}$ is normable. We do not know whether $\widehat{\mathbf{R}}^{\sharp}$ is normable.

We now consider whether the field $\widehat{\mathbf{R}}$ belongs to one of our 'nice' classes. However, it is shown in $[\mathbf{9}$, Chapter 6$]$ that this $\widehat{\mathbf{R}}$ is not even a super-real field, and so maybe $\widehat{\mathbf{R}}$ is not too interesting for our normability question.

To show that a specific field $K$ does not belong to a particular class of fields, one can seek an invariant of the class that $K$ does not possess. The invariant that is used in [9, Chapter 6] is that of 'having an exponentiation'; this is a special case of the theory of an operational calculus for super-real fields, and this latter theory has proved to be important.

The following is [9, Definition 6.15].

Definition 6.5. Let $K$ be an ordered field, and let $I$ be a convex subgroup of $K$. Then an exponentiation on $I$ is an order-isomorphism

$$
\exp : I \rightarrow K^{+} \backslash\{0\}
$$

such that:

(i) $\exp (a+b)=(\exp a)(\exp b) \quad(a, b \in I)$;

(ii) $\exp 0=1$ and $\exp 1=\mathrm{e} 1$.

In the discussion of prime ideals in algebras $C(\Omega)$, we have mentioned some subclasses of the class of all prime ideals. Here is another such class $[\mathbf{9}$, Definition 4.45].

Let $\Omega$ be a compact space. Then a prime ideal $P$ in $C(\Omega)$ is an exponential prime if the function $\exp (-1 / f)$ belongs to $C(\Omega) \backslash P$ whenever $f \in C(\Omega)^{+} \backslash P$. Here $\exp (-1 / f)$ is defined pointwise. Clearly each prime $z$-ideal is an exponential prime; there are exponential primes that are not $z$-ideals and valuation primes that are not exponential primes. The following theorem combines Theorems 6.20, 6.22, and 6.23 of $[\mathbf{9}]$.

TheOREM 6.6. Let $\Omega$ be a compact space, and let $P$ be a prime ideal in $C(\Omega)$. Then there is an exponentiation on a convex subgroup of the super-real field $K_{P}$, and there is an exponentiation on $K_{P}$ itself when $P$ is an exponential prime. However there is no exponentiation on a convex subgroup of the field $\widehat{\mathbf{R}}$, and so $\widehat{\mathbf{R}}$ is not isomorphic as a field to any super-real field.

In particular, the above result shows that there is a real-closed $\eta_{1}$-field that is not a super-real field. A stronger result will be mentioned below. 
6.3. Complete ordered fields. As a preliminary to a discussion of the final field that we wish to introduce, we define complete ordered fields; see [9, Chapter 3]. The next definition is an obvious generalization of the basic concept of completeness for a metric space.

Let $G$ be a totally ordered group, and take an ordinal $\sigma$. Then a sequence $\left(x_{\tau}: \tau<\sigma\right)$ is a Cauchy sequence if, for each $\varepsilon>0$ in $G$, there exists $\sigma_{0}<\sigma$ such that $\left|x_{\tau}-x_{\rho}\right|<\varepsilon$ whenever $\sigma_{0}<\tau<\rho<\sigma$, and the sequence is convergent to $x \in G$ if, for each $\varepsilon>0$ in $G$, there exists $\sigma_{0}<\sigma$ such that $\left|x_{\tau}-x\right|<\varepsilon$ whenever $\sigma_{0}<\tau<\sigma$. The group $G$ is Cauchy complete if each Cauchy sequence of length $\delta(G)$ is convergent to some element of $G$. A completion of $G$ is a totally ordered group $H$ such that $H$ is complete and $G$ is isomorphic to an order-dense subgroup of $H$.

Suppose further that $\delta(G)$ is infinite. Then it is easy to see that $G$ has a unique completion $H$, called the Cauchy completion, and that $H$ is divisble whenever $G$ is divisible. Further $\Gamma_{H}=\Gamma_{G}$, and the archimedean valuation on $H$, when restricted to $G$, agrees with the archimedean valuation on $G$.

The completion of $G$ is denoted by $\widetilde{G}$. We note that $|\widetilde{G}| \leq 2^{w(G)}$.

Here is an example of a completion. Let $S$ be a totally ordered set. Then the divisible group $\mathfrak{F}(S)$ is itself Cauchy complete. Now consider a subgroup $G$ of $\mathfrak{F}(S)$. A function $f \in \mathfrak{F}(S)$ belongs locally to $G$ if, for each $s \in S$, there exists $f_{s} \in G$ such that $f_{s}(t)=f(t)$ for all $t \in S$ with $t \leq s$. In particular, consider $G=\mathfrak{F}_{(1)}(S)$. Then $\widetilde{G}$ is naturally identified with the space of functions in $\mathfrak{F}(S)$ that belong locally to $G$.

Now suppose that $K$ is an ordered field. Then $K$ is Cauchy complete if the totally ordered group $(K,+)$ is Cauchy complete. It is not hard to see that the totally ordered completion $\widetilde{K}$ of the group $(K,+)$ can be made into an ordered field in such a way that $K$ is a subfield of $\widetilde{K}$; in the case that $K$ is real-closed, the field $\widetilde{K}$ is also real-closed. The field $\widetilde{K}$ is the Cauchy completion of $K$.

6.4. The field $\widetilde{\mathbf{R}}$. We finally come to my favourite real-closed field. We define $\widetilde{\mathbf{R}}$ to be the Cauchy completion of $\mathbf{R}$, so that $\widetilde{\mathbf{R}}$ is the space of functions $f$ in $\mathfrak{F}(\mathbf{G})$ that belong locally to $\mathbf{R}=\mathfrak{F}_{(1)}(\mathbf{G})$.

The following theorem is part of [9, Theorem 3.21].

TheOREM 6.7. The field $(\widetilde{\mathbf{R}},+, \cdot, \leq)$ is a real-closed, Cauchy complete $\eta_{1}$-field, with value group $\mathbf{G}$. Further,

$$
|\widetilde{\mathbf{R}}|=2^{\aleph_{1}}, \quad w\left((\widetilde{\mathbf{R}})=\mathfrak{c}, \quad \text { and } \quad \operatorname{cof} \widetilde{\mathbf{R}}=\delta(\widetilde{\mathbf{R}})=\aleph_{1} .\right.
$$

With $(\mathrm{CH}),(\widetilde{\mathbf{R}},+, \cdot, \leq)$ is unique, in the sense that each Cauchy complete $\eta_{1}$-field of weight $\aleph_{1}$ is isomorphic to $(\widetilde{\mathbf{R}},+, \cdot, \leq)$ as an ordered field.

The field $\widetilde{\mathbf{R}}$ is universal in the class of completions of $\beta_{1}$-fields: every completion of a $\beta_{1}$-field is isomorphic to a subfield of $\widetilde{\mathbf{R}}[\mathbf{9}$, Theorem 3.22]. Thus $\widetilde{\mathbf{R}}$ contains every real-closed field of weight $\aleph_{1}$.

Recall that the real line $\mathbb{R}$ is the unique Cauchy complete (equivalently, Dedekind complete) real-closed $\eta_{0}$-field of weight $\aleph_{1}$, and that $|\mathbb{R}|=\aleph_{1}$ with $\mathrm{CH}$. Thus $\widetilde{\mathbf{R}}$ is the natural analogue of $\mathbb{R}$, one cardinality higher, and hence $\widetilde{\mathbf{R}}$ is a 'grown-up' version of the real line. 
We remark that $\widetilde{\mathbf{R}}$ is different from both $\mathbf{R}$ and $\widehat{\mathbf{R}}$. This is immediate on cardinality grounds if we assume $\mathrm{CH}$. However, in general, there is no isotonic map from $\widetilde{\mathbf{R}}$ into $\mathbf{R}$ and no isotonic map from $\widehat{\mathbf{R}}$ into $\widetilde{\mathbf{R}}[\mathbf{9}$, Theorem 3.23$]$, and so $\widetilde{\mathbf{R}}$ is not order-isomorphic to either $\mathbf{R}$ or $\widehat{\mathbf{R}}$.

I would like to show that $\widetilde{\mathbf{R}}^{\sharp}$ is normable, but have not succeeded in this. The field $\widetilde{\mathbf{R}}$ is a test case for Conjecture 6.3 , above: the algebra $K^{\sharp}$ is normable for each ordered field $K$ with $w(K)=\aleph_{1}$ if and only if $\widetilde{\mathbf{R}}^{\sharp}$ is normable.

We now consider whether the field $\widetilde{\mathbf{R}}$ belongs to one of our 'nice' classes. This seems to be much harder to decide than the corresponding question for $\widehat{\mathbf{R}}$.

We showed that $\widehat{\mathbf{R}}$ is not a super-real field by showing that it did not have an exponentiation on any convex subgroup. However this route is not open to us for $\widetilde{\mathbf{R}}$ because there is an exponentiation on $\widetilde{\mathbf{R}}$ itself $[\mathbf{9}$, Theorem 6.24$]$.

The first result that we mention is $[\mathbf{9}$, Theorem 9.6(ii)]: the ordered field $\widetilde{\mathbf{R}}$ is not isomorphic as a field to any ultrapower. Indeed, a somewhat stronger result is given in $[\mathbf{9}$, Theorem 9.9].

Let $\mathcal{U}$ be a free ultrafilter on $\mathbb{N}$, with corresponding ultrapower $\mathbb{R}^{\omega} / \mathcal{U}$. Then $\mathbb{R}^{\omega} / \mathcal{U}$ is not just a real-closed $\eta_{1}$-field of cardinality $\mathfrak{c}$, but it is also a 'non-standard model of analysis': much of the structure of $\mathbb{R}$ can be transfered to $\mathbb{R}^{\omega} / \mathcal{U}$. It is of great importance in [9] that a version of this additional structure exists for each super-real field of the form $K_{P}$. This additional structure is rather straightforward to construct in the case where the prime ideal $P$ is a $z$-ideal because we can work 'pointwise', but it seems to be much more subtle in the case where $P$ is a general prime ideal in an algebra $C(\Omega)$.

A first fruit of this additional structure is a 'gap theorem' [9, Theorem 8.61]. This leads to a deep result [9, Theorem 8.63]: there is a real-closed $\eta_{1}$-field that is not even order-isomorphic to any super-real field.

Study of this additional structure leads to the main theorem of $[\mathbf{9}$, Chapter 9]; it is Theorem 9.26 .

TheOREM 6.8. (GCH) The ordered field $\widetilde{\mathbf{R}}$ is a hyper-real field.

This theorem strengthens the wish to determine whether or not $\widetilde{\mathbf{R}}^{\sharp}$ is normable.

6.5. Conclusions. The final two chapters of $[\mathbf{9}]$ are devoted to showing that the additional structure that has been associated with a super-real field is sometimes an invariant of the field. Thus any classification of super-real fields must involve a prior classification of these structures. In some sense, it is shown that all the structure of these super-real fields is determined just by the order structure of the field. One consequence [9, Theorem 11.49] is that it is relatively consistent with $\mathrm{ZFC}+\mathrm{GCH}$ that there is a super-real field $K_{P}$ that is an $\eta_{1}$-field of weight $\aleph_{1}$ such that $K_{P}$ is not even order-isomorphic to any super-real field $K_{Q}$, where $Q$ is a prime $z$-ideal in some algebra $C(\Omega)$, and hence it cannot be proved in ZFC that all super-real fields are order-isomorphic to a hyper-real field.

A final diagram in [9] considers twelve classes of real-closed $\eta_{1}$-fields, including some classes not mentioned in this article, and eleven classes of super-real fields that are $\eta_{1}$-fields. An aim was to determine, for any two classes, whether one is contained in the other, and whether they are equal; 'contained in' could mean as fields or as totally ordered groups or as ordered sets. We worked in the theory $\mathrm{ZFC}+\mathrm{GCH}$. 
Rather a lot of inclusions are obtained; some inclusions are 'relative consistency' results obtained by forcing. We suspect that there are no more inclusions than the ones that we established; we also suspect that results obtained by forcing are not theorems of ZFC + GCH. There is a list of open questions in Chapter 12 of [9]; we are not aware of any resolution of any of these open questions, and we commend contemplation of them, in particular to those who attended the conference in Harvard.

It will be apparent that the original insights into the theorems of the second half of [9], including all theorems that involve forcing, were due to Hugh Woodin. My comment on his method of proof is the following: first, there were discussions on the likelihood that one class was contained in another class, and possible counterexamples were considered; the questions were clarified and the undergrowth cleared away; then Hugh thought about the matter for some time, and drew a large number of squiggly lines on a sheet of white paper; then he opined, using remarkable insight on what would be true in certain models of set theory, that a particular containment would be relatively consistent with $\mathrm{ZFC}+\mathrm{GCH}$. These insights were almost always completely correct in the end; but it took many days and many pages to craft a careful proof of these insights.

It was a privilege and a pleasure to work with Hugh on these books; I trust that his extraordinary mathematical powers and insights will continue to inspire and to produce beautiful and significant results in the higher reaches of set theory, logic, and the philosophy of mathematics, and maybe even in Banach algebra theory.

\section{References}

[1] G. R. Allan, Embedding the algebra of formal power series in a Banach algebra, Proc. London Math. Soc. (3), 25 (1972), 329-340.

[2] G. R. Allan, Introduction to Banach spaces and algebras, Oxford Graduate Texts in Mathematics, Volume 20, Oxford University Press, 2011.

[3] W. G. Bade and P. C. Curtis, Jr., Homomorphisms of commutative Banach algebras, American J. Math., 82 (1960), 589-608.

[4] W. W. Comfort and S. Negrepontis, The theory of ultrafilters, Springer-Verlag, Berlin, Heidelberg, and New York, 1974.

[5] H. G. Dales, A discontinuous homomorphism from $C(X)$, American J. Math., 101 (1979), $647-734$.

[6] H. G. Dales, Banach algebras and automatic continuity, London Mathematical Society Monographs, Volume 24, Clarendon Press, Oxford, 2000.

[7] H. G. Dales and J. Esterle Discontinuous homomorphisms from $C(X)$, Bulletin of the American Mathematical Society, 83 (1977), 257-259.

[8] H. G. Dales and W. H. Woodin, An introduction to independence for analysts, London Mathematical Society Lecture Note Series 115, Cambridge University Press, 1987.

[9] H. G. Dales and W. H. Woodin, Super-real fields: totally ordered fields with additional structure, London Mathematical Society Monographs, Volume 14, Clarendon Press, Oxford, 1996.

[10] A. Dow, On ultrapowers of Boolean algebras, Topology Procs., 9 (1984), 269-291.

[11] O. Ermert, Continuity of homomorphisms from $A F-C^{*}$-algebras and other inductive limit $C^{*}$-algebras, J. London Math. Soc. (2), 54 (1996), 369-386.

[12] J. R. Esterle, Seminormes sur $\mathcal{C}(K)$, Proc. London Math. Soc. (3), 36 (1978), 27-45.

[13] J. R. Esterle, Injections de semi-groupes divisibles dans des algèbres de convolution et construction d'homomorphismes discontinues de $\mathcal{C}(K)$, Proc. London Math. Soc. (3), 36 (1978), $59-85$. 
[14] J. R. Esterle, Theorems of Gelfand-Mazur type and continuity of epimorphisms from $\mathcal{C}(K)$, J. Functional Aanlysis, 36 (1980), 273-280.

[15] J. R. Esterle, Universal properties of some commutative, radical Banach algebras, J. reine angew. Math., 321 (1981), 1-24.

[16] J. R. Esterle, Remarques sur les théorèmes d'immersion de Hahn et Hausdorff et sur les corps de séries formelles, Quarterly J. Math., Oxford, 51 (2000), 211-219.

[17] L. Gillman and M. Jerison, Rings of continuous functions, van Nostrand Reinhold, New York, 1960; reprinted as Graduate Texts in Mathematics, 43, Springer-Verlag, New York, 1976.

[18] B. E. Johnson, Continuity of homomorphisms of algebras of operators, J. London Math. Soc., 42 (1967), 537-539.

[19] B. E. Johnson, Norming $C(\Omega)$ and related algebras, Trans. American Math. Soc., 220 (1976), $37-58$.

[20] I. Kaplansky, Normed algebras, Duke Math. J., 16 (1949), 399-418.

[21] H. L. Pham, The kernels of radical homomorphisms and intersections of prime ideals, Trans. American Math. Soc., 360 (2008), 1057-1088.

$[22]$ H. L. Pham, Uncountable families of prime $z$-ideals in $\mathcal{C}_{0}(\mathbb{R})$, Bull. London Math. Soc., 41 (2009), 354-366.

[23] H. L. Pham, The kernels and continuity ideals of homomorphisms from $\mathcal{C}_{0}(\Omega)$, J. Australian Math. Soc., 88 (2010), 103-130.

[24] A. M. Sinclair, Homomorphisms from $C^{*}$-algebras, Proc. London Math. Soc. (3), 29 (1974), $435-452$.

[25] A. M. Sinclair, Homomorphisms from $C_{0}(\mathbb{R})$, J. London Math. Soc. (2), 11 (1975), 165-174.

[26] R. M. Solovay and S. Tennenbaum, Iterated Cohen extensions and Souslin's problem, Annals of Math., 94 (1971), 201-245.

[27] S. Todorčević, Partition problems in topology, Contemporary Mathematics, 84, American Mathematical Society, Providence, RI, 1989, xii+116 pp.

[28] W. H. Woodin, Discontinuous homomorphisms from $C(\Omega)$ and the partially ordered set $\omega^{\omega}$, unpublished notes, pp. 24, Caltech, Fall 1976.

[29] W. H. Woodin, Discontinuous homomorphisms of $C(\Omega)$ and set theory, Thesis, University of California, Berkeley, 1984.

[30] W. H. Woodin, A discontinuous homomorphism from $C(X)$ without $\mathrm{CH}, J$. London Math. Soc. (2), 48 (1993), 299-315.

Department of Mathematics and Statistics, University of Lancaster, Lancaster

LA1 4 YF, UK

E-mail address: g.dales@lancaster.ac.uk 